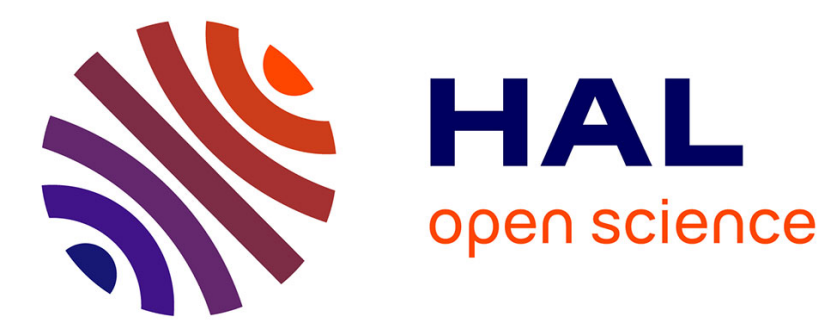

\title{
Discrete variational Lie group formulation of geometrically exact beam dynamics
}

F. Demoures, F. Gay-Balmaz, S. Leyendecker, S. Ober-Blöbaum, T. S. Ratiu, Y. Weinand

\section{- To cite this version:}

F. Demoures, F. Gay-Balmaz, S. Leyendecker, S. Ober-Blöbaum, T. S. Ratiu, et al.. Discrete variational Lie group formulation of geometrically exact beam dynamics. Numerische Mathematik, 2015, 130 (1), pp.73-123. 10.1007/s00211-014-0659-4 . hal-01396186

\section{HAL Id: hal-01396186 \\ https://hal.science/hal-01396186}

Submitted on 14 Nov 2016

HAL is a multi-disciplinary open access archive for the deposit and dissemination of scientific research documents, whether they are published or not. The documents may come from teaching and research institutions in France or abroad, or from public or private research centers.
L'archive ouverte pluridisciplinaire HAL, est destinée au dépôt et à la diffusion de documents scientifiques de niveau recherche, publiés ou non, émanant des établissements d'enseignement et de recherche français ou étrangers, des laboratoires publics ou privés. 


\title{
Discrete variational Lie group formulation of geometrically exact beam dynamics
}

\author{
F. Demoures - F. Gay-Balmaz - S. Leyendecker • \\ S. Ober-Blöbaum • T.S. Ratiu • Y. Weinand
}

\begin{abstract}
The goal of this paper is to derive a structure preserving integrator for geometrically exact beam dynamics, by using a Lie group variational integrator. Both spatial and temporal discretization are implemented in a geometry preserving manner. The resulting scheme preserves both the discrete momentum maps and symplectic structures, and exhibits almost-perfect energy conservation. Comparisons with existing numerical schemes are provided and the convergence behavior is analyzed numerically.
\end{abstract}

\section{Introduction}

The goal of this paper is to derive a structure preserving integrator for geometrically exact beam dynamics formulated on Lie groups. We use the Lagrangian variational

F. Demoures · T. S. Ratiu

Section de Mathématiques, École Polytechnique Fédérale de Lausanne, 1015 Lausanne, Switzerland

F. Gay-Balmaz

Laboratoire de Météorologie Dynamique, CNRS/École Normale Supérieure de Paris, 75005 Paris, France

S. Leyendecker

Department of Mechanical Engineering, University of Erlangen-Nuremberg,

91058 Erlangen, Germany

S. Ober-Blöbaum ( $\varangle)$

Department of Mathematics, University of Paderborn, 33098 Paderborn, Germany

e-mail:sinaob@math.upb.de 
formulation of the continuous problem to obtain a Lie group variational integrator that preserves the symmetries and symplectic structure at the discrete level, and exhibits almost-perfect energy conservation.

The geometrically exact theory of beam dynamics was developed in [59] and [60]. This approach generalizes the formulation originally developed by $[53,54]$ for the plane static problem to the fully 3-dimensional dynamical case. It can be regarded as a convenient parametrization of a three-dimensional extension of the classical Kirchhoff-Love rod model due to [2]. The equations of motion of geometrically exact beams are obtained by applying Hamilton's principle to the Lagrangian (kinetic minus potential energy) defined in material representation and expressed uniquely in terms of convective variables (velocities and strains).

In this paper, we take advantage of this geometric approach to deduce a discrete variational principle in convective representation, thereby obtaining a structure preserving numerical integrator. The discretization is done both spatially and temporally in a manner that preserves the geometric Lie group structure of the problem. Such integrators, based on a discrete version of Hamilton's principle for conservative mechanical systems, are called variational integrators [50]. The associated numerical scheme, given by the discrete Euler-Lagrange equations, is symplectic and momentum-preserving and has an excellent long-time energy behavior. The performance of the newly derived method is illustrated by means of numerical examples including the comparison to other methods, discussing similarities and differences with regard to convergence behavior and computational efficiency.

Based on different variational formulations (e.g., Hamilton, Lagrange-d'Alembert, Hamilton-Pontryagin, etc.), variational integrators have been developed for classical conservative mechanical systems (for an overview see [39,40]), forced [31] and controlled [52] systems, constrained holonomic systems holonomic [43,45] and nonholonomic systems [33], nonsmooth systems [19], stochastic systems [11], and multiscale systems $[44,63]$.

Based on [51], discrete Euler-Lagrange equations for systems on Lie groups, and the associated discrete Lagrangian reductions have been carried out in $[7,8,48]$, and further developed in [37] and applied to many examples. These integrators are referred to as Lie group variational integrators. The essential idea behind such integrators is to discretize Hamiltons principle and to update group elements using group operations.

In the case of the beam, the configuration Lie group is infinite dimensional. It contains the parametrization of the centroid line together with the orientation of crosssections. In order to apply Lie group variational integrators to this case, we first spatially discretize the problem by preserving the Lie group structure.

We also develop an alternative variational integrator for beams, based on the approach used in [10] for systems on Lie groups, following [26]. This approach involves the choice of a retraction map to consistently encode in the Lie algebra the discrete displacement made on the Lie group.

Modeling geometrically exact beams as a special Cosserat continuum (see e.g., [3]) has been the basis for many finite element formulations starting with [59,62]. The formulation of beam dynamics as a Lagrangian system immediately raises the question of the representation of the rotational degrees of freedom and their kinematics, which 
can, on the one hand, be treated by a local parametrization of the the Lie group $S O$ (3) or, on the other hand, by using a redundant configuration variable which is subject to constraints.

Many current semi-discrete beam formulations avoid the introduction of constraints by using rotational degrees of freedom, see, e.g., [25,27]. However, it has been shown by [14], that the interpolation of non-commutative finite rotations bears the risk of destroying the objectivity of the strain measures in the semi-discrete model. This can be circumvented by the introduction of a director triad, which is constrained to be orthonormal in each node of the central line of the beam; thus it forms the columns of the rotational matrix. The spatial interpolation of the director triad leads to objective strain measures in the spatially discretized configuration (even though the interpolated directors might fail to be orthonormal). This idea is independently developed in [55] and [5]. [56] offers an overview on the effects of different interpolation techniques concerning frame invariance and the appearance of singularities. Furthermore, this subject is elaborated in $[4,9,24,28,29]$. The constrained formulation is particularly popular when the beam is part of a multibody system, where further constraints representing the connection to other (rigid or flexible) components are naturally present. Additional popular formulations are the so called absolute nodal coordinates formulation based on works of $[57,58]$ or formulations in terms of unit quaternions [34,35]. Recently, Lie group formulations are becoming more and more important in multibody dynamics; see, e.g., [12,13]. To the authors' knowledge, none of the work on beam dynamics simulation uses a discrete dynamics approach which is variational both in time and in space. However, [30] derives a purely static discrete equilibrium for Cosserat beams using a discrete variational principle in space.

Outline of the paper. In the first part of this paper we review the geometrically exact theory of beams in the continuous setting. In particular, we present the equations of motion via Hamilton's principle which is of central importance for this paper. Then, we explain the Lie group structure underlying the continuous model, and carry out the spatial discretization in a way that preserves both the Lie group structure and the objectivity of the original model. After recalling basic facts about Lie group variational integrators and the associated discrete momentum maps, Legendre transforms and Lagrange-d'Alembert principle, we derive the Lie group variational integrator, and the alternative version based on Lie algebras, for the beam. In particular, we compute the associated discrete momentum maps and consider the effect of external forces at the discrete level. In the last section the performance of the two derived Lie group integrators is demonstrated by two numerical examples. The first example is a standard benchmark problem consisting of a beam with concentrated masses at the middle and the boundary nodes and additional external forces. The simulation results are verified based on a comparison with the discrete solution obtained by an energy-momentum preserving time stepping scheme with finite elements in space [41]. Based on a second example simulating the dynamics of an initially deformed beam, a numerical convergence analysis is performed for all three methods.

Before closing this Introduction, we recall below some basic facts concerning Lagrangian mechanics, both at the continuous and discrete levels. 
Lagrangian dynamics and momentum maps. For a given a Lagrangian $L: T Q \rightarrow \mathbb{R}$ defined on the tangent bundle $T Q$ of the configuration manifold $Q$, the Euler-Lagrange equations are obtained by applying Hamilton's principle to the action functional associated to $L$. Symmetries are given by Lie group actions $\Phi: G \times Q \rightarrow Q$, $(g, q) \mapsto \Phi_{g}(q)$ under which the Lagrangian is invariant. In this case, the Noether theorem guarantees that the associated momentum map $\mathbf{J}: T^{*} Q \rightarrow \mathfrak{g}^{*}$, given by

$$
\left\langle\mathbf{J}\left(\alpha_{q}\right), \xi\right\rangle=\left\langle\alpha_{q}, \xi_{Q}(q)\right\rangle \quad \alpha_{q} \in T^{*} Q, \xi \in \mathfrak{g}
$$

see [1], is a conserved quantity, where $\mathfrak{g}$ denotes the Lie algebra of the Lie group $G$, $\mathfrak{g}^{*}$ its dual, and the vector field $\xi_{Q}$ on $Q$ is the infinitesimal generator of the action associated to $\xi \in \mathfrak{g}$, that is,

$$
\xi_{Q}(q):=\left.\frac{d}{d \varepsilon}\right|_{\varepsilon=0} \Phi_{\exp (\varepsilon \xi)}(q),
$$

where exp : $\mathfrak{g} \rightarrow G$ is the exponential map of the Lie group $G$.

In presence of an external force field, given by a fiber preserving map $F: T Q \rightarrow$ $T^{*} Q$, Hamilton's principle is replaced by the Lagrange-d'Alembert principle (see e.g., [49])

$$
\delta \int_{0}^{T} L(q(t), \dot{q}(t)) d t+\int_{0}^{T} F(q(t), \dot{q}(t)) \cdot \delta q d t=0,
$$

where $F(q, \dot{q}) \cdot \delta q$ is the virtual work done by the force field $F$ with a virtual displacement $\delta q$. This principle yields the Lagrange-d'Alembert equations

$$
\frac{d}{d t} \frac{\partial L}{\partial \dot{q}}-\frac{\partial L}{\partial q}=F(q, \dot{q})
$$

Discrete Lagrange-d'Alembert principle. A complete presentation of discrete Lagrangian mechanics, discrete momentum maps, and discrete Noether theorems is given in [50]. We shall now review some of these aspects, by mainly focusing on the discrete Lagrange-d'Alembert principle.

Suppose that a time step $\Delta t$ has been fixed, denote by $\left\{t^{j}=j \Delta t \mid j=0, \ldots, N\right\}$ the sequence of time, and by $q_{d}:\left\{t^{j}\right\}_{j=0}^{N} \rightarrow Q, q_{d}\left(t^{j}\right)=q^{j}$ a discrete curve. Let $L_{d}: Q \times Q \rightarrow \mathbb{R}, L_{d}=L_{d}\left(q^{j}, q^{j+1}\right)$ be a discrete Lagrangian which we think of as approximating the action integral of $L$ along the curve segment between $q^{j}$ and $q^{j+1}$. The discrete Lagrange-d'Alembert principle seeks discrete curves $\left\{q^{j}\right\}_{j=0}^{N}$ that satisfy

$$
\delta \sum_{j=0}^{N-1} L_{d}\left(q^{j}, q^{j+1}\right)+\sum_{j=0}^{N-1}\left(F_{d}^{-}\left(q^{j}, q^{j+1}\right) \cdot \delta q^{j}+F_{d}^{+}\left(q^{j}, q^{j+1}\right) \cdot \delta q^{j+1}\right)=0
$$

for variations $\delta q^{j}$ vanishing at endpoints, where the two discrete Lagrangian forces $F_{d}^{ \pm}: Q \times Q \rightarrow T^{*} Q$ are fiber preserving maps such that the second term above is 
an approximation of the integral $\int_{0}^{T} F(q, \dot{q}) \cdot \delta q d t$ of the virtual work. One gets the discrete Euler-Lagrange equations with forces

$$
D_{1} L_{d}\left(q^{j}, q^{j+1}\right)+D_{2} L_{d}\left(q^{j-1}, q^{j}\right)+F_{d}^{+}\left(q^{j-1}, q^{j}\right)+F_{d}^{-}\left(q^{j}, q^{j+1}\right)=0,
$$

for $j=1, \ldots, N-1$. In the forced case, the discrete Legendre transforms are defined by

$$
\begin{aligned}
& \mathbb{F}^{F+} L_{d}\left(q^{j}, q^{j+1}\right):=D_{2} L_{d}\left(q^{j}, q^{j+1}\right)+F_{d}^{+}\left(q^{j}, q^{j+1}\right) \in T_{q^{j+1}}^{*} Q \\
& \mathbb{F}^{F-} L_{d}\left(q^{j}, q^{j+1}\right):=-D_{1} L_{d}\left(q^{j}, q^{j+1}\right)-F_{d}^{-}\left(q^{j}, q^{j+1}\right) \in T_{q^{j}}^{*} Q .
\end{aligned}
$$

The discrete Euler-Lagrange equations with forces can be equivalently written as

$$
\mathbb{F}^{F+} L_{d}\left(q^{j-1}, q^{j}\right)=\mathbb{F}^{F-} L_{d}\left(q^{j}, q^{j+1}\right), \text { for } j=1, \ldots, N-1 .
$$

Given the discrete forces $F^{ \pm}$, and a Lie group action $\Phi: G \times Q \rightarrow Q$, the discrete Lagrangian momentum maps $\mathbf{J}_{L_{d}}^{F^{+}}, \mathbf{J}_{L_{d}}^{F^{-}}: Q \times Q \rightarrow \mathfrak{g}^{*}$ are defined by

$$
\begin{aligned}
& \left\langle\mathbf{J}_{L_{d}}^{F^{+}}\left(q^{j}, q^{j+1}\right), \xi\right\rangle=\left\langle D_{2} L_{d}\left(q^{j}, q^{j+1}\right)+F^{+}\left(q^{j}, q^{j+1}\right), \xi_{Q}\left(q^{j+1}\right)\right\rangle \\
& \left\langle\mathbf{J}_{L_{d}}^{F^{-}}\left(q^{j}, q^{j+1}\right), \xi\right\rangle=\left\langle-D_{1} L_{d}\left(q^{j}, q^{j+1}\right)-F^{-}\left(q^{j}, q^{j+1}\right), \xi_{Q}\left(q^{j}\right)\right\rangle .
\end{aligned}
$$

Note that if the discrete curve $\left\{q^{j}\right\}_{j=0}^{N}$ verifies the discrete Euler-Lagrange equations with forces (3) then we have the equality

$$
\mathbf{J}_{L_{d}}^{F^{+}}\left(q^{j-1}, q^{j}\right)=\mathbf{J}_{L_{d}}^{F^{-}}\left(q^{j}, q^{j+1}\right), \quad \text { for all } j=1, \ldots, N-1
$$

If the discrete Lagrangian $L_{d}$ is $G$-invariant under the action $\Phi Q \times Q$ of $G$ on $Q \times Q$, and if $F_{d}^{-}\left(q^{j}, q^{j+1}\right) \cdot \xi_{Q}\left(q^{j}\right)+F_{d}^{+}\left(q^{j}, q^{j+1}\right) \cdot \xi_{Q}\left(q^{j+1}\right)=0$ then the two discrete momentum maps coincide, $\mathbf{J}_{L_{d}}^{F^{-}}=\mathbf{J}_{L_{d}}^{F^{+}}=: \mathbf{J}_{L_{d}}^{F}$, therefore from (6), we obtain that $\mathbf{J}_{L_{d}}^{F}$ is a conserved quantity along the discrete curve solution of (3), that is,

$$
\mathbf{J}_{L_{d}}^{F}\left(q^{j}, q^{j+1}\right)=\mathbf{J}_{L_{d}}^{F}\left(q^{j-1}, q^{j}\right), \quad \text { for all } j=1, \ldots, N-1 .
$$

This result is referred to as the discrete forced Noether's theorem.

Approximate energy conservation. In absence of forcing, the main feature of the numerical scheme $\left(q^{j-1}, q^{j}\right) \mapsto\left(q^{j}, q^{j+1}\right)$ given by solving the discrete EulerLagrange equations is that the associated scheme $\left(q^{j}, p^{j}\right) \mapsto\left(q^{j+1}, p^{j+1}\right)$ induced on the phase space $T^{*} Q$ through the discrete Legendre transform defines a symplectic integrator. Here we supposed that the discrete Lagrangian $L_{d}$ is regular, that is, both discrete Legendre transforms $\mathbb{F}^{+} L_{d}, \mathbb{F}^{-} L_{d}: Q \times Q \rightarrow T^{*} Q$ are locally isomorphisms (for nearby $q^{j}$ and $q^{j+1}$ ). The symplectic character of the integrator is obtained by showing that the scheme $\left(q^{j-1}, q^{j}\right) \mapsto\left(q^{j}, q^{j+1}\right)$ preserves the discrete 
symplectic two-forms $\Omega_{L_{d}}^{ \pm}:=\left(\mathbb{F}^{ \pm} L_{d}\right)^{*} \Omega_{\text {can }}$, where $\Omega_{\text {can }}$ is the canonical symplectic form on $T^{*} Q$, so that $\left(q^{j}, p^{j}\right) \mapsto\left(q^{j+1}, p^{j+1}\right)$ preserves $\Omega_{\text {can }}$ and is therefore symplectic, see $[39,50]$.

It is known (see [23]), that given a Hamiltonian $H$, a symplectic integrator for $H$ is exactly solving a modified Hamiltonian system for a Hamiltonian $\bar{H}$ which is close to $H$. So the discrete trajectory has all the properties of a conservative Hamiltonian system, such as conservation of the energy $\bar{H}$. The same conclusion holds on the Lagrangian side for variational integrators (see, e.g., [39]). This explains why energy is approximately conserved for variational integrators, and typically oscillates about the true energy. We refer to [23] for a detailed account and a full treatment of backward error analysis for symplectic integrators.

\section{Lagrangian dynamics of a beam in $\mathbb{R}^{3}$}

\subsection{Basic kinematics of a beam}

We review below the kinematic description of a beam in the ambient space $\mathbb{R}^{3}$ following [59], see also [60]. The static version of the beam model summarized below goes back essentially to [54] who modified the classical Kirchhoff-Love model to account for shear deformation.

The configuration of a beam is defined by specifying the position of its line of centroids by means of a map $\phi:[0, L] \rightarrow \mathbb{R}^{3}$ and the orientation of cross-sections at points $\phi(S)$ by means of a moving basis $\left\{\mathbf{d}_{1}(S), \mathbf{d}_{2}(S), \mathbf{d}_{3}(S)\right\}$ (sometimes called directors) attached to the cross section. The orientation of the moving basis is described with the help of an orthogonal transformation $\Lambda:[0, L] \rightarrow S O(3)$ such that $\mathbf{d}_{I}(S)=$ $\Lambda(S) \mathbf{E}_{I}, I=1,2,3$, where $\left\{\mathbf{E}_{1}, \mathbf{E}_{2}, \mathbf{E}_{3}\right\}$ is a fixed basis referred to as the material frame. The configuration of the beam is thus completely determined by the maps $\phi$ and $\Lambda$ in the configuration space

$$
\mathbf{Q}=C^{\infty}\left([0, L], S O(3) \times \mathbb{R}^{3}\right) \ni \Phi=(\Lambda, \phi)
$$

If boundary conditions are imposed, then they need to be included in this configuration space. For example at $S=0$, one can consider the boundary conditions $\phi(0)=$ $0, \Lambda(0)=I d$, that is, the point $\phi(0)$ of the line of centroid is fixed (e.g. stays at the origin) and the cross-section at the point $\phi(0)$ is fixed. One can also impose the condition $\phi^{\prime}(0)=\lambda \mathbf{E}_{3}$, for an arbitrary $\lambda>0$, which means that the line of centroid at $\phi(0)$ is orthogonal to the plan defined by $\mathbf{E}_{1}$ and $\mathbf{E}_{2}$. At the other extremity $S=L$ similar boundaries conditions can be imposed.

The time-evolution of the beam is described by a curve $\Phi(t)=(\Lambda(t), \phi(t)) \in \mathbf{Q}$, in the configuration space. The material velocity $V_{\Phi}$ is defined by

$$
V_{\Phi}(S, t)=\frac{d}{d t} \Phi(S, t)=(\dot{\Lambda}(S, t), \dot{\phi}(S, t))
$$


and thus belongs to the tangent space $T_{\Phi} \mathbf{Q}$ to $\mathbf{Q}$ at $\Phi$. Before defining the convective angular and linear velocities, we first recall some notations concerning the Lie algebra of $S O$ (3).

Notations. We denote by $\mathfrak{s o}(3)$ the Lie algebra of $S O(3)$ consisting of skew symmetric matrices endowed with the Lie bracket $[A, B]=A B-B A$. Throughout the paper we use the standard Lie algebra isomorphism $\widehat{ }{ }^{-}\left(\mathbb{R}^{3}, \times\right) \rightarrow(\mathfrak{s o}(3),[]$,$) , called the$ hat map and defined by

$$
\Omega=\left(\begin{array}{c}
\Omega^{1} \\
\Omega^{2} \\
\Omega^{3}
\end{array}\right) \mapsto \widehat{\Omega}:=\left(\begin{array}{ccc}
0 & -\Omega^{3} & \Omega^{2} \\
\Omega^{3} & 0 & -\Omega^{1} \\
-\Omega^{2} & \Omega^{1} & 0
\end{array}\right)
$$

We have the identity $\operatorname{Ad}_{A} \widehat{\Omega}=\widehat{A \Omega}$.

2.2 Lagrangian of the beam

The Lagrangian $L: T \mathbf{Q} \rightarrow \mathbb{R}$ of the geometrically exact beam has the expression

$$
\begin{aligned}
L(\Lambda, \phi, \dot{\Lambda}, \dot{\phi})= & \frac{1}{2} \int_{0}^{L}\left(M\|\gamma\|^{2}+\omega^{T} J \omega\right) d S \\
& -\int_{0}^{L} \Psi_{i n t}(\Omega, \Gamma) d S-\int_{0}^{L}\langle\mathbf{q}, \phi\rangle d S,
\end{aligned}
$$

where

$$
\widehat{\omega}=\Lambda^{T} \dot{\Lambda} \text { and } \gamma=\Lambda^{T} \dot{\phi}
$$

are the convected angular velocity and convected linear velocity, respectively, and

$$
\widehat{\Omega}=\Lambda^{T} \Lambda^{\prime} \text { and } \Gamma=\Lambda^{T} \phi^{\prime}
$$

are the convected angular deformation gradient and convected linear deformation gradient, respectively.

The first integral in (9) represents the kinetic energy of the beam and is obtained by integrating the kinetic energy of the material points over the whole body. It is assumed that the mass density is a constant $\rho_{0}$ and that the mid-line $\phi$ passes through the center of mass of the cross section, given by a compact subset $\mathcal{A} \subset \mathbb{R}^{2}$ with smooth boundary. The constant $M=\rho_{0} \times \operatorname{area}(\mathcal{A})$ is the distributed loads per unit length, and $J$ is the inertia matrix in the body fixed frame defined as

$$
J:=-\int_{\mathcal{A}} \rho_{0}\left(\xi^{1} \widehat{\mathbf{E}_{1}+\xi^{2}} \mathbf{E}_{2}\right)^{2} d \xi^{1} d \xi^{2}
$$


The rotational part of the kinetic energy can also we written in terms of $\widehat{\omega}$ as

$$
\frac{1}{2} \int_{0}^{L} \operatorname{Tr}\left(\widehat{\omega}^{T} J_{d} \widehat{\omega}\right) d S, \quad \text { where } J_{d}:=\int_{\mathcal{A}} \rho_{0}\left(\xi^{1} \mathbf{E}_{1}+\xi^{2} \mathbf{E}_{2}\right)\left(\xi^{1} \mathbf{E}_{1}+\xi^{2} \mathbf{E}_{2}\right)^{T} d \xi^{1} d \xi^{2}
$$

is the (non-standard) diagonal inertia matrix, also called Euler tensor.

The second term in (9) is the bending potential energy, written in terms of the stored energy function $\Psi_{\text {int }}$. We assume that the unstressed state is undeformed, that is, we have $\phi^{\prime}(S, t=0)=\mathbf{E}_{3}$ and $\Lambda(S, t=0)=I d$, for all $S \in[0, L]$. With these assumptions, the stored energy reads

$$
\Pi_{i n t}(\Lambda, \phi)=\frac{1}{2} \int_{0}^{L}\left[\left(\Gamma-\mathbf{E}_{3}\right)^{T} \mathbf{C}_{1}\left(\Gamma-\mathbf{E}_{3}\right)+\Omega^{T} \mathbf{C}_{2} \Omega\right] d S
$$

where we defined the matrices

$$
\mathbf{C}_{1}:=\operatorname{Diag}\left(G A_{1} G A_{2} \quad E A\right) \text { and } \mathbf{C}_{2}:=\operatorname{Diag}\left(E I_{1} \quad E I_{2} \quad G J\right)
$$

of elasticity coefficients. Here $A_{1}=A_{2}=A=\operatorname{area}(\mathcal{A})$ is the cross-sectional area of the rod, $I_{1}$ and $I_{2}$ are the principal moments of inertia of the cross-section, $J=I_{1}+I_{2}$ is its polar moment of inertia, $E$ is Young's modulus, $G=E /[2(1+v)]$ is the shear modulus, and $v$ is Poisson's ratio. This internal energy is used in [61] and [17], taking into account that the thickness of the rod is small compared to its length, and that the material is homogeneous and isotropic.

The third term in (9) is the exterior potential energy created by a conservative force q per unit length.

Note that the first two terms of the Lagrangian are invariant under the left action of the group $S E(3)$, given by $(\Lambda, \phi) \mapsto(R, \mathbf{v})(\Lambda, \phi)=(R \Lambda, \mathbf{v}+R \phi)$.

Stresses. The stresses along the beam are defined by

$$
\mathbf{n}:=\frac{\partial \Psi_{i n t}}{\partial \Gamma}=\mathbf{C}_{1}\left(\Gamma-\mathbf{E}_{3}\right)
$$

where the $\mathbf{E}_{1}$ - and $\mathbf{E}_{2}$-components are the shear stresses and the $\mathbf{E}_{3}$-component is the stretch stress. The momenta along the beam are defined by

$$
\mathbf{m}:=\frac{\partial \Psi_{\text {int }}}{\partial \Omega}=\mathbf{C}_{2} \Omega
$$

where the $\mathbf{E}_{1}$ - and $\mathbf{E}_{2}$-components are the bending momenta, with respect to the principal axes of the cross-section, and the $\mathbf{E}_{3}$-component is the torsional moment.

\subsection{Equations of motion}

The equations of motion are derived by applying the by computing the Lagranged'Alembert principle to the Lagrangian (9) and with given external forces $F: T \mathbf{Q} \rightarrow$ 
$T^{*} \mathbf{Q}, F=(\mathfrak{M}, \mathfrak{F})$. They read

$$
\left\{\begin{array}{l}
J \dot{\omega}+\omega \times J \omega+\mathbf{C}_{1}\left(\Gamma-\mathbf{E}_{3}\right) \times \Gamma-\Omega \times \mathbf{C}_{2} \Omega-\mathbf{C}_{2} \Omega^{\prime}=\Lambda^{-1} \mathfrak{M} \\
M \ddot{\phi}-\left(\Lambda \mathbf{C}_{1}\left(\Gamma-\mathbf{E}_{3}\right)\right)^{\prime}+\mathbf{q}=\mathfrak{F}
\end{array}\right.
$$

with boundary conditions

$$
\left.\left(\Gamma-\mathbf{E}_{3}\right)\right|_{S=0}=0,\left.\quad\left(\Gamma-\mathbf{E}_{3}\right)\right|_{S=L}=0, \quad \Omega(0)=\Omega(L)=0 .
$$

Here the condition $\left.\left(\Gamma-\mathbf{E}_{3}\right)\right|_{S=0}=0$ means that the mid-line remains orthogonal to the cross section at point $\phi(0)$ at all time. The condition $\Omega(0)=0$ means there is no bending nor torsion at the end boundary. Note that different kinds of forcing is possible like dead loads, configuration-dependent follower forces or velocity-dependent dissipative forces.

We note that, if we remove the forces, the equations (14) can be written as follows

$$
\left\{\begin{array}{l}
J \dot{\omega}+\omega \times J \omega+\mathbf{n} \times \Gamma-\Omega \times \mathbf{m}-\mathbf{m}^{\prime}=0 \\
M \ddot{\phi}-\Lambda(\Omega \times \mathbf{n})-\Lambda \mathbf{n}^{\prime}+\mathbf{q}=0
\end{array}\right.
$$

where $\mathbf{m}$ and $\mathbf{n}$ are the stresses and the momenta as defined in (12) and (13). These are the statements of balance of angular, mass and linear momentum in the convective description, as in [60]. We refer to [18] and [20] for more details concerning the Lagrangian reduction process involved in the formulation of geometrically exact rods and to [46] and [21] for the geometric description of the convective representation in nonlinear elasticity and its associated Lagrangian variational formulation.

\section{Lie group variational integrator for the beam}

\subsection{The Lie group structure}

The goal of this section is to develop a Lie group variational integrator for the beam. This can be done by identifying the configuration space $\mathbf{Q}$ of the beam with the infinite dimensional Lie group $G=C^{\infty}([0, L], S E(3))$, with group multiplication given by pointwise multiplication in the group $S E(3)$, that is $\left(\Lambda_{1}, \phi_{1}\right)\left(\Lambda_{2}, \phi_{2}\right)=$ $\left(\Lambda_{1} \Lambda_{2}, \phi_{1}+\Lambda_{1} \phi_{2}\right)$. The convective velocity can be written as

$$
(\hat{\omega}, \gamma)=(\Lambda, \phi)^{-1}(\dot{\Lambda}, \dot{\phi})
$$

It is important to observe that, in this setting, if boundary conditions have to be imposed on the configuration space, they have to preserve the group structure. For example, both boundary conditions considered in Fig. 1 preserve the group structure of $G$. 


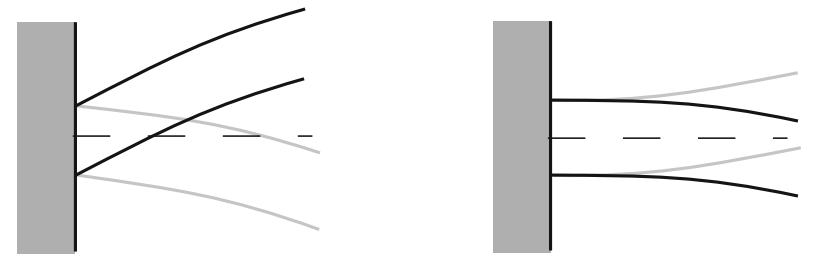

Fig. 1 Illustration of the boundary conditions: $\Lambda(0)=I d, \phi(0)=0($ left $)$, and $\Lambda(0)=I d, \phi(0)=$ $0, \phi^{\prime}(0)=\mathbf{E}_{3}($ right $)$

\subsection{Spatial discretization}

Discretization of the variables. We decompose the interval $[0, L]$ into $A$ subintervals $K=\left[S_{a}, S_{a+1}\right]$ of length $l_{K}=S_{a+1}-S_{a}$. We denote by $\mathcal{T}$ the set of all elements $K$ that subdivide the interval $[0, L]$. The configuration of the beam at the node $a$ is given by $\Lambda_{a}:=\Lambda\left(S_{a}\right)$ and $\mathbf{x}_{a}=\phi\left(S_{a}\right)$.

Given the configurations $\left(\Lambda_{a}, \mathbf{x}_{a}\right)$ and $\left(\Lambda_{a+1}, \mathbf{x}_{a+1}\right)$ of the beam at the nodes $a$ and $a+1$, we consider the following interpolations over the subinterval $K$

$$
\left.\Lambda_{h}(S)\right|_{K}:=\Lambda_{a} \exp \left(\frac{S}{l_{K}} \widehat{\psi}_{a}\right) \text { and }\left.\phi_{h}(S)\right|_{K}:=\mathbf{x}_{a}+\frac{S}{l_{K}} \Delta \mathbf{x}_{a}
$$

where $S \in\left[0, l_{K}\right], \Delta \mathbf{x}_{a}:=\mathbf{x}_{a+1}-\mathbf{x}_{a}$ and $\exp \left(\widehat{\psi}_{a}\right):=\Lambda_{a}^{T} \Lambda_{a+1}$. Note that we consistently have $\Lambda_{h}\left(l_{K}\right)=\Lambda_{a+1}$ and $\mathbf{x}_{h}\left(l_{K}\right)=\mathbf{x}_{a+1}$. This interpolation was considered by [14] in order to obtain a spatial discretization that preserves the objectivity of the strain measures $\Omega$ and $\Gamma .{ }^{1}$ Note that, for simplicity, we parametrize the element $K$ using $S \in\left[0, l_{K}\right]$ instead of $S \in\left[S_{a}, S_{a+1}\right]$.

The associated convected variables $\widehat{\omega}_{h}(S), \gamma_{h}(S), \widehat{\Omega}_{h}(S)$, and $\Gamma_{h}(S)$ are obtained by using the approximations $\phi_{h}(S)$ and $\Lambda_{h}(S)$ instead of the original variables $\phi(S)$ and $\Lambda(S)$ in their definitions. We thus get

$$
\begin{aligned}
\widehat{\omega}_{h}(S) & =\Lambda_{h}(S)^{T} \dot{\Lambda}_{h}(S), \\
\gamma_{h}(S) & =\Lambda_{h}(S)^{T} \dot{\phi}_{h}(S)=\Lambda_{h}(S)^{T}\left(\dot{\mathbf{x}}_{a}+S \Delta \dot{\mathbf{x}}_{a} / l_{K}\right), \\
\widehat{\Omega}_{h}(S) & =\Lambda_{h}(S)^{T} \Lambda_{h}^{\prime}(S)=\widehat{\psi}_{a} / l_{K}, \\
\Gamma_{h}(S) & =\Lambda_{h}(S)^{T} \phi_{h}^{\prime}(S)=\Lambda_{h}(S)^{T} \Delta \mathbf{x}_{a} / l_{K} .
\end{aligned}
$$

Note that by considering $S=0$ and $S=l_{K}$, we obtain that at each node the relation (15) between the material and convected velocities is preserved, that is, $\omega_{a}=\Lambda_{a}^{T} \dot{\Lambda}_{a}$, $\omega_{a+1}=\Lambda_{a+1}^{T} \dot{\Lambda}_{a+1}$, and $\gamma_{a}=\Lambda_{a}^{T} \dot{\mathbf{x}}_{a}, \quad \gamma_{a+1}=\Lambda_{a+1}^{T} \dot{\mathbf{x}}_{a+1}$.

We use the notation $\Lambda_{K}=\left(\Lambda_{a}, \Lambda_{a+1}\right)^{T}, \mathbf{x}_{K}=\left(\mathbf{x}_{a}, \mathbf{x}_{a+1}\right)^{T}$, and similarly for $\dot{\Lambda}_{K}, \dot{\mathbf{x}}_{K}, \omega_{K}, \gamma_{K}$, to denote the variables associated to an element $K$ with nodes $a$ and $a+1$.

1 Consider a superposed rigid motion in $(\Lambda, \phi)$ given by the transformation $(\tilde{\Lambda}, \widetilde{\phi})=(R \Lambda, \mathbf{v}+R \phi)$, where $R \in S O(3)$, and $\mathbf{v} \in \mathbb{R}^{3}$. Since $\left(\widetilde{\Lambda}_{a}\right)^{T} \widetilde{\Lambda}_{a+1}=\Lambda_{a}^{T} \Lambda_{a+1}$ and $\widetilde{\Lambda}_{a}^{T} \Delta \widetilde{\mathbf{x}}_{a}=\Lambda_{a}^{T} \Delta \mathbf{x}_{a}$, the strain measures are unchanged by this transformation. 
The boundary conditions in Fig. 1 are given by $\Lambda_{a_{0}}=I d, \mathbf{x}_{a_{0}}=0$, or $\Lambda_{a_{0}}=I d$, $\mathbf{x}_{a_{0}}=0, \Delta \mathbf{x}_{a_{0}}=\lambda \mathbf{E}_{3}, \lambda>0$.

The discrete Lagrangian. The spatially discretized Lagrangian is obtained by inserting the variables considered in (17) and $\Lambda_{h}, \Phi_{h}$ in the continuous Lagrangian (9) and by considering the trapezoidal rule approximations.

For the kinetic energy, we make the following approximations on an element $K$ of length $l_{K}$ :

$$
\begin{aligned}
\frac{1}{2} \int_{0}^{l_{K}} M\left\|\gamma_{h}(S)\right\|^{2} d S & \approx \frac{l_{K}}{4} M\left(\left\|\gamma_{a}\right\|^{2}+\left\|\gamma_{a+1}\right\|^{2}\right), \\
\frac{1}{2} \int_{0}^{l_{K}}\left(\omega_{h}(S)^{T} J \omega_{h}(S)\right) d S & \approx \frac{l_{K}}{4}\left(\omega_{a}^{T} J \omega_{a}+\omega_{a+1}^{T} J \omega_{a+1}\right) .
\end{aligned}
$$

Concerning the potential energy, the expression obtained by using $\Lambda_{h}$ and $\phi_{h}$ instead of $\Lambda$ and $\phi$ is denoted by

$$
V_{K}\left(\Lambda_{K}, \mathbf{x}_{K}\right):=\int_{K} \mathcal{V}_{h}(S) d S,
$$

where $\mathcal{V}_{h}(S):=\Psi_{\text {int }}\left(\Lambda_{h}(S), \phi_{h}(S)\right)+\Psi_{\text {ext }}\left(\phi_{h}(S)\right)$. We approximate the potential energy $V_{K}$ with the expression $\mathbb{V}_{K}$ defined by

$$
\begin{aligned}
\mathbb{V}_{K}\left(\Lambda_{K}, \mathbf{x}_{K}\right):=\frac{l_{K}}{2}\left(\mathcal{V}_{h}(0)+\mathcal{V}_{h}\left(l_{K}\right)\right) \\
=\frac{l_{K}}{4}\left[\left(\Lambda_{a}^{T} \frac{\Delta \mathbf{x}_{a}}{l_{K}}-\mathbf{E}_{3}\right)^{T} \mathbf{C}_{1}\left(\Lambda_{a}^{T} \frac{\Delta \mathbf{x}_{a}}{l_{K}}-\mathbf{E}_{3}\right)\right. \\
\left.\quad+\left(\Lambda_{a+1}^{T} \frac{\Delta \mathbf{x}_{a}}{l_{K}}-\mathbf{E}_{3}\right)^{T} \mathbf{C}_{1}\left(\Lambda_{a+1}^{T} \frac{\Delta \mathbf{x}_{a}}{l_{K}}-\mathbf{E}_{3}\right)+\frac{2}{\left(l_{K}\right)^{2}}\left(\psi_{a}\right)^{T} \mathbf{C}_{2} \psi_{a}\right] \\
\quad+\frac{l_{K}}{2}\left\langle\mathbf{q}_{a}, \mathbf{x}_{a}\right\rangle+\frac{l_{K}}{2}\left\langle\mathbf{q}_{a+1}, \mathbf{x}_{a+1}\right\rangle,
\end{aligned}
$$

where we recall that $\Delta \mathbf{x}_{a}=\mathbf{x}_{a+1}-\mathbf{x}_{a}$, and $\widehat{\psi}_{a}=\exp ^{-1}\left(\Lambda_{a}^{T} \Lambda_{a+1}\right)$. In this last expression, the exponential map can be approximated by the Cayley transform cay : $\mathfrak{g} \rightarrow G$ defined by

$$
\Lambda=\operatorname{cay}(\widehat{\Omega})=(I-\widehat{\Omega} / 2)^{-1}(I+\widehat{\Omega} / 2)
$$

with inverse $\widehat{\Omega}=\operatorname{cay}^{-1}(\Lambda)=2(\Lambda-I)(\Lambda+I)^{-1}$.

As a consequence, the spatially discretized Lagrangian $L_{K}: \operatorname{TSE}(3)^{2} \rightarrow \mathbb{R}$ over an element $K$ of length $l_{K}$, is given by

$$
L_{K}\left(\Lambda_{K}, \mathbf{x}_{K}, \dot{\Lambda}_{K}, \dot{\mathbf{x}}_{K}\right)=\frac{l_{K}}{4} M\left(\left\|\dot{\mathbf{x}}_{a}\right\|^{2}+\left\|\dot{\mathbf{x}}_{a+1}\right\|^{2}\right)
$$




$$
+\frac{l_{K}}{4}\left(\omega_{a}^{T} J \omega_{a}+\omega_{a+1}^{T} J \omega_{a+1}\right)-\mathbb{V}_{K}\left(\mathbf{x}_{K}, \Lambda_{K}\right)
$$

The spatial discrete Lagrangian $L$ of the total system is obtained by summing over all the elements $K$, that is $L=\sum_{K \in \mathcal{T}} L_{K}$. Assuming that all elements $K$ have initially the same undeformed length $l_{K}$ and taking care of boundary nodes $a=0$ and $a=A$, we get

$$
\begin{aligned}
L\left(\left(\Lambda_{a}, \mathbf{x}_{a}, \dot{\Lambda}_{a}, \dot{\mathbf{x}}_{a}\right)_{a \in \mathcal{N}}\right)= & \sum_{a \in \operatorname{int}(\mathcal{N})}\left(\frac{l_{K}}{2} M\left\|\dot{\mathbf{x}}_{a}\right\|^{2}+\frac{l_{K}}{2} \omega_{a}^{T} J \omega_{a}\right) \\
& +\sum_{a \in \partial \mathcal{N}}\left(\frac{l_{K}}{4} M\left\|\dot{\mathbf{x}}_{a}\right\|^{2}+\frac{l_{K}}{4} \omega_{a}^{T} J \omega_{a}\right) \\
& -\sum_{K \in \mathcal{T}} \mathbb{V}_{K}\left(\mathbf{x}_{K}, \Lambda_{K}\right),
\end{aligned}
$$

where $\mathcal{N}$ denotes the set of all nodes, $\partial \mathcal{N}=\{0, A\}$ is the set of boundary nodes, and $\operatorname{int}(\mathcal{N})=\{1, \ldots, A-1\}$ denotes the set of internal nodes.

Discrete stresses. Similar with (12) and (13), the discrete stresses along the beam are defined by $\mathbf{n}_{h}:=\frac{\partial \mathcal{V}_{h}}{\partial \Gamma_{h}}=\mathbf{C}_{1}\left(\Gamma_{h}-\mathbf{E}_{3}\right)$. Given an element $K$, the associated discrete stress is defined by

$\mathbf{n}_{K}:=\frac{1}{2}\left(\mathbf{n}_{h}(0)+\mathbf{n}_{h}\left(l_{K}\right)\right)=\frac{1}{2}\left(\mathbf{C}_{1}\left(\Lambda_{a} \frac{\Delta \mathbf{x}_{a}}{l_{K}}-\mathbf{E}_{3}\right)+\mathbf{C}_{1}\left(\Lambda_{a+1} \frac{\Delta \mathbf{x}_{a}}{l_{K}}-\mathbf{E}_{3}\right)\right)$.

The discrete momenta along the beam are defined by $\mathbf{m}_{h}:=\frac{\partial \mathcal{V}_{h}}{\partial \Omega_{h}}=\mathbf{C}_{2} \Omega_{h}$. As before, the discrete momenta associated with $K$ read

$$
\mathbf{m}_{K}:=\frac{1}{2}\left(\mathbf{m}_{h}(0)+\mathbf{m}_{h}\left(l_{K}\right)\right)=\mathbf{C}_{2} \psi_{a} / l_{K}
$$

\subsection{Lie group variational integrators}

\subsubsection{Discrete Lagrange-d'Alembert equations on Lie groups}

Lie group variational integrators, originated in the work of [51], were developed in $[7,8,48]$ for the numerical treatment of mechanical systems on finite dimensional Lie groups, by using a discrete analogue of Lagrangian reduction. These methods were further developed and exploited in [37]. In this approach, given a Lagrangian $L: T G \rightarrow \mathbb{R}$ defined on the tangent bundle $T G$ of a Lie group $G$, the discrete trivialized Lagrangian $\mathcal{L}_{d}\left(g^{j}, f^{j}\right): G \times G \rightarrow \mathbb{R}$ is defined such that the following approximation holds: 


$$
\mathcal{L}_{d}\left(g^{j}, f^{j}\right) \approx \int_{t^{j}}^{t^{j+1}} L(g(t), \dot{g}(t)) d t
$$

where $g(t)$ is the solution of the Euler-Lagrange equations with $g\left(t^{j}\right)=g^{j}$ and $g\left(t^{j+1}\right)=g^{j+1}=g^{j} f^{j}$. For simplicity we use the notation $\mathcal{L}_{d}^{j}:=\mathcal{L}_{d}\left(g^{j}, f^{j}\right)$.

As recalled in the Introduction, the discrete Lagrange-d'Alembert principle is formulated with the help of discrete Lagrangian forces $F_{d}^{ \pm}=F^{ \pm}\left(g^{j}, g^{j+1}\right): G \times G \rightarrow$ $T^{*} G$. In the case of Lie group variational integrators, we reformulate $F_{d}^{ \pm}$in terms of $g^{j}$ and $f^{j}$ as follows. We define the discrete forces $\mathcal{F}_{d}^{ \pm}: G \times G \rightarrow T^{*} G$ by $\mathcal{F}_{d}^{-}\left(g^{i}, f^{i}\right):=F_{d}^{-}\left(g^{j}, g^{j+1}\right) \in T_{g^{j}}^{*} G$ and $\mathcal{F}_{d}^{+}\left(g^{i+1}, f^{i}\right):=F_{d}^{+}\left(g^{j}, g^{j+1}\right) \in$ $T_{g j+1}^{*} G$, where $g^{j+1}=g^{j} f^{j}$. From (2) and using these definitions, we deduce that the discrete Lagrange-d'Alembert principle for Lie group variational integrators is

$$
\delta \sum_{j=0}^{N-1} \mathcal{L}_{d}\left(g^{j}, f^{j}\right)+\sum_{j=0}^{N-1}\left[\mathcal{F}_{d}^{-}\left(g^{j}, f^{j}\right) \cdot \delta g^{j}+\mathcal{F}_{d}^{+}\left(g^{j+1}, f^{j}\right) \cdot \delta g^{j+1}\right]=0,
$$

for all variations $\delta g^{j}$ vanishing at endpoints. The variational principle $\delta \mathfrak{S}_{d}=0$ yields the discrete Lagrange-d'Alembert equations

$$
\begin{aligned}
& \left(g^{j}\right)^{-1} D_{g^{j}} \mathcal{L}_{d}^{j}-\operatorname{Ad}_{\left(f^{j}\right)^{-1}}^{*}\left(\left(f^{j}\right)^{-1} D_{f^{j}} \mathcal{L}_{d}^{j}\right)+\left(f^{j-1}\right)^{-1} D_{f^{j-1}} \mathcal{L}_{d}^{j-1} \\
& \quad+\left(g^{j}\right)^{-1} \mathcal{F}_{d}^{+}\left(g^{j}, f^{j-1}\right)+\left(g^{j}\right)^{-1} \mathcal{F}_{d}^{-}\left(g^{j}, f^{j}\right)=0 \\
& \quad \text { with } g^{j}=g^{j-1} f^{j-1}
\end{aligned}
$$

see Proposition 3.2 in [7]. Note that given $\left(g^{j-1}, f^{j-1}\right)$, we obtain $g^{j}=g^{j-1} f^{j-1}$ from the second equation, and we solve the first equation to find $f^{j}$. This yields a discrete-time flow map $\left(g^{j-1}, f^{j-1}\right) \in G \times G \mapsto\left(g^{j}, f^{j}\right) \in G \times G, j=1, \ldots, N$.

Notations for Lie groups. Left and right multiplications by $g \in G$ are denoted by $L_{g}, R_{g}: G \rightarrow G, L_{g}(f)=g f, R_{g}(f)=f g$. The tangent lifted actions $T L_{g}, T R_{g}$ : $T G \rightarrow T G$ are sometimes denoted as $g v_{f}:=T L_{g}\left(v_{f}\right)$ and $v_{f} g:=T R_{g}\left(v_{f}\right)$ for simplicity, where $v_{f} \in T G$. Similarly, the cotangent lifted actions $T^{*} L_{g^{-1}}, T^{*} R_{g^{-1}}$ : $T^{*} G \rightarrow T^{*} G$ is denoted by $g \alpha_{f}:=T^{*} L_{g^{-1}}\left(\alpha_{f}\right)$ and $\alpha_{f} g:=T^{*} R_{g^{-1}}\left(\alpha_{f}\right), \alpha_{f} \in$ $T^{*} G$, for simplicity. This notation is used in (24).

Discrete Legendre transforms. Recall that, in the forced case, there are two discrete Legendre transforms $\mathbb{F}^{F \pm} L_{d}: G \times G \rightarrow T^{*} G$ associated to a discrete Lagrangian $L_{d}\left(g^{j}, g^{j+1}\right)$, see (4). We write the Legendre transforms in terms of the discrete Lagrangian $\mathcal{L}_{d}\left(g^{j}, f^{j}\right)$ used for Lie group variational integrators, by using the following relation between $L_{d}$ and $\mathcal{L}_{d}$, namely,

$$
\mathcal{L}_{d}\left(g^{j}, f^{j}\right)=L_{d}\left(g^{j}, g^{j+1}\right) \quad \text { with } g^{j+1}=g^{j} f^{j} .
$$


From (4), we obtain that the forced discrete Legendre transforms $\mathbb{F}^{\mathcal{F} \pm} \mathcal{L}_{d}: G \times G \rightarrow$ $G \times \mathfrak{g}^{*}$ are

$$
\mathbb{F}^{\mathcal{F}+} \mathcal{L}_{d}^{j}=\left(g^{j+1},\left(\pi_{\mathcal{F}}^{j}\right)^{+}\right) \quad \text { and } \quad \mathbb{F}^{\mathcal{F}-} \mathcal{L}_{d}^{j}=\left(g^{j},\left(\pi_{\mathcal{F}}^{j}\right)^{-}\right)
$$

where $\pi_{\mathcal{F}_{ \pm}}^{j}$ are the discrete body momenta in presence of forces defined by

$$
\begin{aligned}
\left(\pi_{\mathcal{F}}^{j}\right)^{+} & :=\left(\pi^{j}\right)^{+}+\left(g^{j} f^{j}\right)^{-1} \mathcal{F}_{d}^{+}\left(g^{j} f^{j}, f^{j}\right) \\
= & \left(f^{j}\right)^{-1} D_{f^{j}} \mathcal{L}_{d}^{j}+\left(g^{j} f^{j}\right)^{-1} \mathcal{F}_{d}^{+}\left(g^{j} f^{j}, f^{j}\right) \\
\left(\pi_{\mathcal{F}}^{j}\right)^{-} & :=\left(\pi^{j}\right)^{-}-\left(g^{j}\right)^{-1} \mathcal{F}_{d}^{-}\left(g^{j}, f^{j}\right) \\
= & \left.-\left(g^{j}\right)^{-1} D_{g^{j}} \mathcal{L}_{d}^{j}+\operatorname{Ad}_{\left(f^{j}\right)^{-1}}^{*}\left(\left(f^{j}\right)^{-1} D_{f^{j}} \mathcal{L}_{d}^{j}\right)\right) \\
& -\left(g^{j}\right)^{-1} \mathcal{F}_{d}^{-}\left(g^{j}, f^{j}\right) .
\end{aligned}
$$

The discrete Lagrange-d'Alembert equations can be equivalently written as

$$
\mathbb{F}^{\mathcal{F}+} \mathcal{L}_{d}^{j-1}=\mathbb{F}^{\mathcal{F}-} \mathcal{L}_{d}^{j}, \quad \text { i.e. } \quad g^{j-1} f^{j-1}=g^{j} \quad \text { and } \quad\left(\pi_{\mathcal{F}}^{j-1}\right)^{+}=\left(\pi_{\mathcal{F}}^{j}\right)^{-} .
$$

Discrete momentum mappings and subgroup actions. Recall from (5) that, given the discrete forces $F^{ \pm}$, a discrete Lagrangian $L_{d}\left(q^{j}, q^{j+1}\right)$ and a Lie group action of $G$ on $Q$, two discrete momentum maps $\mathbf{J}_{L_{d}}^{F^{ \pm}}: Q \times Q \rightarrow \mathfrak{g}^{*}$ can be defined. In the present case, we shall choose a subgroup $H$ of $G$ and consider the action of $H$ on $G$ by left translation. Using the relation (25) and the expression (5), we get

$$
\begin{gathered}
\mathbf{J}_{\mathcal{L}_{d}^{+}}^{F^{+}}\left(g^{j}, f^{j}\right)=i^{*}\left(\operatorname{Ad}_{\left(g^{j+1}\right)^{-1}}^{*}\left(\left(f^{j}\right)^{-1} D_{f^{j}} \mathcal{L}_{d}^{j}\right)+\operatorname{Ad}_{\left(g^{j+1}\right)^{-1}}^{*}\left(\left(g^{j} f^{j}\right)^{-1} \mathcal{F}_{d}^{+}\left(g^{j} f^{j}, f^{j}\right)\right)\right), \\
\mathbf{J}_{\mathcal{L}_{d}}^{F^{-}}\left(g^{j}, f^{j}\right)=i^{*}\left(-\operatorname{Ad}_{\left(g^{j}\right)^{-1}}^{*}\left(\left(g^{j}\right)^{-1} D_{g^{j}} \mathcal{L}_{d}^{j}\right)+\operatorname{Ad}_{\left(g^{j+1}\right)^{-1}}^{*}\left(\left(f^{j}\right)^{-1} D_{f^{j}} \mathcal{L}_{d}^{j}\right)\right. \\
\left.-\operatorname{Ad}_{\left(g^{j}\right)^{-1}}^{*}\left(\left(g^{j}\right)^{-1} \mathcal{F}_{d}^{-}\left(g^{j}, f^{j}\right)\right)\right),
\end{gathered}
$$

where $i^{*}: \mathfrak{g}^{*} \rightarrow \mathfrak{h}^{*}$ is the dual map to the Lie algebra inclusion $i: \mathfrak{h} \rightarrow \mathfrak{g}$. We note that we have the relations

$\mathbf{J}_{\mathcal{L}_{d}}^{F^{+}}\left(g^{j}, f^{j}\right)=i^{*}\left(\operatorname{Ad}_{\left(g^{j+1}\right)^{-1}}^{*}\left(\pi_{\mathcal{F}}^{j}\right)^{+}\right) \quad$ and $\quad \mathbf{J}_{\mathcal{L}_{d}^{-}}^{F^{-}}\left(g^{j}, f^{j}\right)=i^{*}\left(\operatorname{Ad}_{\left(g^{j}\right)^{-1}}^{*}\left(\pi_{\mathcal{F}}^{j}\right)^{-}\right)$

between the discrete momentum maps and the discrete Legendre transforms and that the discrete Lagrange-d'Alembert equations imply the relation

$$
\mathbf{J}_{\mathcal{L}_{d}}^{F^{+}}\left(g^{j-1}, f^{j-1}\right)=\mathbf{J}_{\mathcal{L}_{d}}^{F^{-}}\left(g^{j}, f^{j}\right)
$$

in $\mathfrak{h}^{*}$. The quantities $\operatorname{Ad}_{\left(g^{j+1}\right)^{-1}}^{*}\left(\left(\pi_{\mathcal{F}}^{j}\right)^{+}\right)$and $\operatorname{Ad}_{\left(g^{j}\right)^{-1}}^{*}\left(\left(\pi_{\mathcal{F}}^{j}\right)^{-}\right)$are referred to as the discrete spatial momenta in presence of forces. 
We note that in the special case $H=G$, the relation (29) is not only implied by the discrete Lagrange-d'Alembert equations, but is equivalent to them.

If the discrete Lagrangian $\mathcal{L}_{d}$ is $H$-invariant and if the discrete forces are orthogonal to the group action, then the two momentum maps coincide: $\mathbf{J}_{\mathcal{L}_{d}^{+}}^{F^{+}}=\mathbf{J}_{\mathcal{L}_{d}}^{F^{-}}=: \mathbf{J}_{\mathcal{L}_{d}}^{F}$, and (29) yields the discrete forced Noether theorem

$$
\mathbf{J}_{\mathcal{L}_{d}}^{F}\left(g^{j-1}, f^{j-1}\right)=\mathbf{J}_{\mathcal{L}_{d}}^{F}\left(g^{j}, f^{j}\right)
$$

Example $G=S E$ (3). We compute the relation (28) for the Lie group $S E$ (3) because of its importance in beam dynamics. We identify the Lie algebra $\mathfrak{s e}(3)=\mathfrak{s o}(3) \subseteq \mathbb{R}^{3}$ of $S E$ (3) with $\mathbb{R}^{3} \times \mathbb{R}^{3}$ by using the hat map (8). Via this identification, the adjoint action reads $\operatorname{Ad}_{(\Lambda, \phi)}(\mathbf{u}, \mathbf{v})=(\Lambda \mathbf{u}, \Lambda \mathbf{v}+\phi \times \Lambda \mathbf{u})$. Identifying the dual space $\mathfrak{s e}(3)^{*}$ with $\mathbb{R}^{3} \times \mathbb{R}^{3}$ via the usual pairing on $\mathbb{R}^{3}$, the coadjoint action reads

$$
\operatorname{Ad}_{(\Lambda, \phi)^{-1}}^{*}(\mathbf{m}, \mathbf{n})=(\Lambda \mathbf{m}+\phi \times \Lambda \mathbf{n}, \Lambda \mathbf{n}) .
$$

The discrete body momenta $\left(\pi_{\mathcal{F}}^{j}\right)^{ \pm} \operatorname{read}\left(\pi_{\mathcal{F}}^{j}\right)^{-}=\left(\left(\Pi_{\mathcal{F}}^{j}\right)^{-},\left(\Gamma_{\mathcal{F}}^{j}\right)^{-}\right)$and $\left(\pi_{\mathcal{F}}^{j}\right)^{+}=$ $\left(\left(\Pi_{\mathcal{F}}^{j}\right)^{+},\left(\Gamma_{\mathcal{F}}^{j}\right)^{+}\right)$, where $\left(\Pi_{\mathcal{F}}^{j}\right)^{ \pm}$are the discrete angular momenta and $\left(\Gamma_{\mathcal{F}}^{j}\right)^{ \pm}$are the discrete linear momenta. Using the notations $g^{j}=\left(\Lambda^{j}, \mathbf{x}^{j}\right), f^{j}=\left(F^{j}, H^{j}\right) \in$ $S E(3)$, the relations (28) read

$$
\begin{aligned}
\mathbf{J}_{\mathcal{L}_{d}^{+}}^{F^{+}}\left(\left(\Lambda^{j}, \mathbf{x}^{j}\right),\left(F^{j}, H^{j}\right)\right) & =\operatorname{Ad}_{\left(\Lambda^{j+1}, \mathbf{x}^{j+1}\right)^{-1}}^{*}\left(\left(\Pi_{\mathcal{F}}^{j}\right)^{+},\left(\Gamma^{j}\right)_{\mathcal{F}}^{+}\right) \\
& =\left(\Lambda^{j+1}\left(\Pi_{\mathcal{F}}^{j}\right)^{+}+\mathbf{x}^{j+1} \times \Lambda^{j+1}\left(\Gamma_{\mathcal{F}}^{j}\right)^{+}, \Lambda^{j+1}\left(\Gamma_{\mathcal{F}}^{j}\right)^{+}\right), \\
\mathbf{J}_{\mathcal{L}_{d}}^{F^{-}}\left(\left(\Lambda^{j}, \mathbf{x}^{j}\right),\left(F^{j}, H^{j}\right)\right) & =\operatorname{Ad}_{\left(\Lambda^{j}, \mathbf{x}^{j}\right)^{-1}}^{*}\left(\left(\Pi_{\mathcal{F}}^{j}\right)^{-},\left(\Gamma_{\mathcal{F}}^{j}\right)^{-}\right) \\
& =\left(\Lambda^{j}\left(\Pi_{\mathcal{F}}^{j}\right)^{-}+\mathbf{x}^{j} \times \Lambda^{j}\left(\Gamma_{\mathcal{F}}^{j}\right)^{-}, \Lambda^{j}\left(\Gamma_{\mathcal{F}}^{j}\right)^{-}\right) .
\end{aligned}
$$

\subsubsection{Symplecticity of the properties of the discrete flow, without forces}

As we already recalled, the numerical scheme $\left(g^{j-1}, g^{j}\right) \mapsto\left(g^{j}, g^{j+1}\right)$ given by the discrete Euler-Lagrange equations yields a symplectic integrator $\left(g^{j}, p^{j}\right) \mapsto$ $\left(g^{j+1}, p^{j+1}\right)$ on $T^{*} G$ by using the discrete Legendre transforms $\mathbb{F}^{ \pm} L_{d}\left(g^{j}, g^{j+1}\right)$. This implies the same property for Lie group variational integrators, namely, the numerical scheme $\left(g^{j}, \mu^{j}\right) \mapsto\left(g^{j+1}, \mu^{j+1}\right)$ induced on $G \times \mathfrak{g}^{*}$ by using the discrete Legendre transforms $\mathbb{F}^{ \pm} \mathcal{L}_{d}\left(g^{j}, f^{j}\right)$ is symplectic relative to the trivialized canonical symplectic form on $G \times \mathfrak{g}^{*}$, see [7,48].

As a consequence, the trivialized Hamiltonian $h: T^{*} G \simeq G \times \mathfrak{g}^{*} \rightarrow \mathbb{R}$ obtained from the continuous Lagrangian $L: T G \rightarrow \mathbb{R}$ by Legendre transform is approximately conserved, that is, the sequence $h\left(g^{j}, \mu^{j}\right), j=0, \ldots, N$ oscillates about the true value of the Hamiltonian.

Remark 1 As in the continuous case, the discrete symplectic form will not be preserved in the presence of forcing. 


\subsection{Alternative discretization on Lie groups}

In this subsection we present a variational integrator for mechanics on Lie groups based on $[10,26,32]$. We will later apply this variational integrator to the beam. This integrator is obtained by choosing a local diffeomorphism $\tau: \mathfrak{g} \rightarrow G$ in a neighborhood of the origin and such that $\tau(0)=e$. Examples for $\tau$ are provided by the exponential map or the Cayley transform. The approach will involve the right trivialized derivative $\mathrm{d}^{R} \tau$ of $\tau$ defined by

$$
\mathrm{d}^{R} \tau(\xi): \mathfrak{g} \rightarrow \mathfrak{g}, \quad \mathrm{d}^{R} \tau(\xi)(\eta):=\left(T_{\xi} \tau(\eta)\right) \tau(\xi)^{-1},
$$

where $T_{\xi} \tau: \mathfrak{g} \rightarrow T_{\tau(\xi)} G$ is the derivative of $\tau$. The right trivialized derivative of $\tau^{-1}$ is defined by d ${ }^{R} \tau^{-1}(\xi)(\eta)=T_{g} \tau^{-1}(\eta g)$, where $g:=\tau(\xi)$. It is readily checked that $\mathrm{d}^{R} \tau^{-1}(\xi)=\left(\mathrm{d}^{R} \tau(\xi)\right)^{-1}$. For example, this right trivialized derivative of $\tau$ was used in [10] to develop a variety of integrators of variational partitioned Runge-Kutta type for Lie groups.

Let $L: T G \rightarrow \mathbb{R}$ be a Lagrangian defined on the tangent bundle $T G$ of a Lie group $G$. Let $\mathscr{L}_{d}: G \times \mathfrak{g} \rightarrow \mathbb{R}$ be a discrete Lagrangian defined as an approximation of the action functional over one time step, namely, we have

$$
\mathscr{L}_{d}\left(g^{j}, \xi^{j}\right) \approx \int_{t^{j}}^{t^{j+1}} L(g(t), \dot{g}(t)) d t
$$

where $g(t)$ is the unique solution of the Euler-Lagrange equations such that $g\left(t^{j}\right)=g^{j}$ and $g\left(t^{j+1}\right)=g^{j+1}$ and where

$$
\tau\left(\Delta t \xi^{i}\right)=\left(g^{j}\right)^{-1} g^{j+1} .
$$

We assume that the time step is small enough so that $\left(g^{j}\right)^{-1} g^{j+1}$ is in a neighborhood of the identity element of $G$ where the map $\tau$ is a diffeomorphism.

In terms of the discrete variables $\left(g^{j}, \xi^{j}\right)$, the discrete Lagrange-d'Alembert principle (2) reads

$$
\delta \sum_{j=0}^{N-1} \mathscr{L}_{d}\left(g^{j}, \xi^{j}\right)+\sum_{j=0}^{N-1}\left[\mathcal{F}_{d}^{-}\left(g^{j}, \xi^{j}\right) \cdot \delta g^{j}+\mathcal{F}_{d}^{+}\left(g^{j+1}, \xi^{j}\right) \cdot \delta g^{j+1}\right]=0
$$

for all variations $\delta g^{j}$ with $\delta g^{0}=\delta g^{N}=0$, where $\mathcal{F}^{-}\left(g^{j}, \xi^{j}\right) \in T_{g^{j}}^{*} G$ and $\mathcal{F}^{+}\left(g^{j+1}, \xi^{j}\right) \in T_{g^{j+1}}^{*} G$ are the discrete external Lagrangian forces. These discrete forces are chosen in such a way that the second term in the variational principle is an approximation of the virtual work done by the force field in the continuous case. Using the notation $\eta^{j}=\left(g^{j}\right)^{-1} \delta g^{j}$, we have the variations

$$
\delta g^{j+1}=g^{j}\left(\operatorname{Ad}_{\tau\left(\Delta t \xi^{j}\right)} \eta^{j+1}\right) \tau\left(\Delta t \xi^{j}\right)
$$




$$
\Delta t \delta \xi^{j}=\mathrm{d}^{R} \tau^{-1}\left(\Delta t \xi^{j}\right)\left(-\eta^{j}+\operatorname{Ad}_{\tau\left(\Delta t \xi^{j}\right)} \eta^{j+1}\right) .
$$

Then, since $\eta^{0}=\eta^{N}=0$, the discrete variational principle $\delta \mathfrak{S}_{d}=0$ yields the discrete Lagrange d'Alembert equations

$$
\left\{\begin{array}{c}
\mu^{j}-\operatorname{Ad}_{\tau\left(\Delta t \xi^{j-1}\right)}^{*} \mu^{j-1}=\left(g^{j}\right)^{-1}\left(D_{g j} c L_{d}\left(g^{j}, \xi^{j}\right)\right)+\left(g^{j}\right)^{-1}\left(\mathcal{F}_{d}^{-}\left(g^{j}, \xi^{j}\right)\right) \\
+\left(g^{j}\right)^{-1}\left(\mathcal{F}_{d}^{+}\left(g^{j}, \xi^{j-1}\right)\right) \\
\mu^{j}=\frac{1}{\Delta t}\left(\mathrm{~d}^{R} \tau^{-1}\left(\Delta t \xi^{j}\right)\right)^{*}\left(D_{\xi^{j}} \mathscr{L}_{d}\left(g^{j}, \xi^{j}\right)\right), \quad g^{j+1}=g^{j} \tau\left(\Delta t \xi^{j}\right),
\end{array}\right.
$$

where $\left(\mathrm{d}^{R} \tau^{-1}(\xi)\right)^{*}: \mathfrak{g}^{*} \rightarrow \mathfrak{g}^{*}$ denotes the dual map to $\mathrm{d}^{R} \tau^{-1}(\xi): \mathfrak{g} \rightarrow \mathfrak{g}$.

Since forces are present, one has to incorporate them in the discrete Legendre transforms and the discrete momentum maps, as in (4). In terms of the discrete variables $\left(g^{j}, \xi^{j}\right)$, the forced discrete Legendre transforms $\mathbb{F}^{f \pm} L_{d}: G \times \mathfrak{g} \rightarrow G \times \mathfrak{g}^{*}$ are

$$
\begin{aligned}
& \mathbb{F}^{f+} L_{d}\left(g^{j}, \xi^{j}\right)=\left(g^{j+1}, \operatorname{Ad}_{\tau\left(\Delta t \xi^{j}\right)}^{*} \mu^{j}+\left(g^{j+1}\right)^{-1}\left(\mathcal{F}_{d}^{+}\left(g^{j+1}, \xi^{j}\right)\right)\right) \\
& \mathbb{F}^{f-} L_{d}\left(g^{j}, \xi^{j}\right)=\left(g^{j}, \mu^{j}-\left(g^{j}\right)^{-1} D_{g j} \mathscr{L}_{d}\left(g^{j}, \xi^{j}\right)-\left(g^{j}\right)^{-1} \mathcal{F}_{d}^{-}\left(g^{j}, \xi^{j}\right)\right) .
\end{aligned}
$$

Let $H \subset G$ be a subgroup, with Lie algebra $\mathfrak{h}$, and consider its action on $G$ by left translation. The forced discrete Lagrangian momentum maps $J_{\mathscr{L}_{d}}^{f \pm}: G \times \mathfrak{g} \rightarrow \mathfrak{h}^{*}$, for $g^{j} \in G$ and $\xi^{j} \in \mathfrak{g}$, are computed by using the formula (5), where one has to express it in terms of the discrete variables $\left(g^{j}, \xi^{j}\right)$ instead of $\left(g^{j}, g^{j+1}\right)$. We obtain

$$
\begin{aligned}
& J_{\mathscr{L}_{d}}^{f+}\left(g^{j}, \xi^{j}\right)=i^{*} \operatorname{Ad}_{\left(g^{j+1}\right)^{-1}}^{*}\left(\operatorname{Ad}_{\tau\left(\Delta t \xi^{j}\right)}^{*} \mu^{j}+\left(g^{j+1}\right)^{-1}\left(\mathcal{F}_{d}^{+}\left(g^{j+1}, \xi^{j}\right)\right)\right), \\
& J_{\mathscr{L}_{d}}^{f-}\left(g^{j}, \xi^{j}\right)=i^{*} \operatorname{Ad}_{\left(g^{j}\right)^{-1}}^{*}\left(\mu^{j}-\left(g^{j}\right)^{-1} D_{g^{j}} \mathscr{L}_{d}\left(g^{j}, \xi^{j}\right)-\left(g^{j}\right)^{-1} \mathcal{F}_{d}^{-}\left(g^{j}, \xi^{j}\right)\right),
\end{aligned}
$$

where $i^{*}: \mathfrak{g}^{*} \rightarrow \mathfrak{h}^{*}$ denotes the dual map to the Lie algebra inclusion $i: \mathfrak{h} \rightarrow \mathfrak{g}$. Note that discrete the forced Euler-Lagrange equations (35) imply the equality

$$
J_{\mathscr{L}_{d}}^{f+}\left(g^{j-1}, \xi^{j-1}\right)=J_{\mathscr{L}_{d}}^{f-}\left(g^{j}, \xi^{j}\right)
$$

If the discrete Lagrangian $\mathscr{L}_{d}$ is $H$-invariant and if the discrete forces verify the condition $\left\langle\mathcal{F}^{-}\left(g^{j}, \xi^{j}\right), \eta g^{j}\right\rangle+\left\langle\mathcal{F}^{+}\left(g^{j+1}, \xi^{j}\right), \eta g^{j+1}\right\rangle=0$, for all $\eta \in \mathfrak{h}$, then the two discrete momentum maps coincide: $J_{\mathscr{L}_{d}}^{f-}=J_{\mathscr{L}_{d}}^{f+}=: J_{\mathscr{L}_{d}}$. Therefore, by (37), we obtain the discrete forced Noether's theorem

$$
J \mathscr{L}_{d}\left(g^{j}, \xi^{j}\right)=J_{\mathscr{L}_{d}}\left(g^{j-1}, \xi^{j-1}\right), \quad \text { for all } j=1, \ldots, N-1
$$

as in (7). 
Remark 2 (Discrete body momenta) Recall that for mechanical systems on Lie groups the spatial and body momenta associated to a momentum $\alpha_{g}=\partial L / \partial \dot{g} \in T^{*} G$ are respectively given by $\pi_{S}=\alpha_{g} g^{-1}$ and $\pi_{B}=g^{-1} \alpha_{g}$, so that we can write $\pi_{B}=$ $\operatorname{Ad}_{g}^{*} \pi_{S}$. Note also that the momentum map associated to left invariance reads $\mathbf{J}_{L}$ : $T^{*} G \rightarrow \mathfrak{g}^{*}, \mathbf{J}_{L}\left(\alpha_{g}\right)=\alpha_{g} g^{-1}$ and thus coincides with the spatial momentum so that we have $\pi_{B}=\operatorname{Ad}_{g}^{*} \mathbf{J}_{L}\left(\alpha_{g}\right)$.

At the discrete level, the discrete body momenta are defined by analogous formulas, namely $\pi_{B}^{ \pm}\left(g^{j}, \xi^{j}\right)=\operatorname{Ad}_{g j}^{*} J_{\mathscr{L}_{d}}^{ \pm}\left(g^{j}, \xi^{j}\right)$, and the discrete Euler-Lagrange equations (35) can be written in terms of $\pi_{B}^{ \pm}$as

$$
\pi_{B}^{+}\left(g^{j-1}, \xi^{j-1}\right)+\left(g^{j}\right)^{-1} \mathcal{F}_{d}^{+}\left(g^{j}, \xi^{j-1}\right)=\pi_{B}^{-}\left(g^{j}, \xi^{j}\right)-\left(g^{j}\right)^{-1} \mathcal{F}_{d}\left(g^{j}, \xi^{j}\right) .
$$

3.5 Lie group variational integrator for the beam

\subsubsection{Time discretization}

Using the same notation as before, given a node $a$, the discrete time evolution of this node is given by the discrete curve $\left(\Lambda_{a}^{j}, \mathbf{x}_{a}^{j}\right), j=0, \ldots, N$ in $S E$ (3). The discrete variables $g^{j}$ and $f^{j}=\left(g^{j}\right)^{-1} g^{j+1}$ associated to this node are $\left(\Lambda_{a}^{j}, \mathbf{x}_{a}^{j}\right)$ and $\left(F_{a}^{j}, H_{a}^{j}\right):=$ $\left(\Lambda_{a}^{j}, \mathbf{x}_{a}^{j}\right)^{T}\left(\Lambda_{a}^{j+1}, \mathbf{x}_{a}^{j+1}\right)=\left(\left(\Lambda_{a}^{j}\right)^{T} \Lambda_{a}^{j+1},\left(\Lambda_{a}^{j}\right)^{T}\left(\mathbf{x}_{a}^{j+1}-\mathbf{x}_{a}^{j}\right)\right) \in S O(3) \times \mathbb{R}^{3}$, where, in the last equality, we used multiplication in $S E(3)$. We denote the time-step by $\Delta t=t^{j}-t^{j-1}$, supposed to be of uniform length.

In terms of these variables $\left(F_{a}^{j}, H_{a}^{j}\right)$, we make the following approximations:

$$
\begin{aligned}
& \widehat{\omega}_{a}^{j}=\left(\Lambda_{a}^{j}\right)^{T} \dot{\Lambda}_{a}^{j} \approx\left(\Lambda_{a}^{j}\right)^{T}\left(\frac{\Lambda_{a}^{j+1}-\Lambda_{a}^{j}}{\Delta t}\right)=\frac{F_{a}^{j}-I_{3}}{\Delta t} \\
& \gamma_{a}^{j}=\left(\Lambda_{a}^{j}\right)^{T} \dot{\mathbf{x}}_{a}^{j} \approx\left(\Lambda_{a}^{j}\right)^{T}\left(\frac{\mathbf{x}_{a}^{j+1}-\mathbf{x}_{a}^{j}}{\Delta t}\right)=\frac{H_{a}^{j}}{\Delta t} .
\end{aligned}
$$

With this approximation, the kinetic energy due to rotation, at $a \in K$, is

$$
\begin{aligned}
\frac{l_{K} \Delta t}{4} \operatorname{Tr}\left(\widehat{\omega}_{a}^{j} J_{d}\left(\widehat{\omega}_{a}^{j}\right)^{T}\right) & \approx \frac{l_{K}}{4 \Delta t} \operatorname{Tr}\left(\left(F_{a}^{j}-I_{3}\right) J_{d}\left(F_{a}^{j}-I_{3}\right)^{T}\right) \\
& =\frac{l_{K}}{2 \Delta t} \operatorname{Tr}\left(\left(I_{3}-F_{a}^{j}\right) J_{d}\right) .
\end{aligned}
$$

The discrete Lagrangian $L_{K}^{j}$ approximating the action of the Lagrangian $L_{K}$ in (20) during the time step $\Delta t$ is therefore

$$
\mathcal{L}_{K}^{j}=\sum_{a \in K}\left\{\frac{l_{K}}{4} \frac{M\left\|H_{a}^{j}\right\|^{2}}{\Delta t}+\frac{l_{K}}{2} \frac{\operatorname{Tr}\left(\left(I_{3}-F_{a}^{j}\right) J_{d}\right)}{\Delta t}\right\}-\Delta t \mathbb{V}_{K}\left(\Lambda_{K}^{j}, \mathbf{x}_{K}^{j}\right)
$$


The discrete action sum, which approximates the continuous action over the time interval $[0, T]$, is computed as follows

$$
\begin{aligned}
\mathfrak{S}_{d}\left(\left(\Lambda_{d}, \mathbf{x}_{d}\right)\right)= & \sum_{K \in \mathcal{T}} \sum_{1 \leq j<N} \mathcal{L}_{K}^{j} \\
= & \sum_{a \neq 0, A} \sum_{j=0}^{N-1}\left\{\frac{l_{K}}{2} \frac{M\left\|H_{a}^{j}\right\|^{2}}{\Delta t}+l_{K} \frac{\operatorname{Tr}\left(\left(I_{3}-F_{a}^{j}\right) J_{d}\right)}{\Delta t}\right\} \\
& +\sum_{j=0}^{N-1}\left\{\frac{l_{K}}{4} \frac{M\left\|H_{0}^{j}\right\|^{2}}{\Delta t}+\frac{l_{K}}{2} \frac{\operatorname{Tr}\left(\left(I_{3}-F_{0}^{j}\right) J_{d}\right)}{\Delta t}\right\} \\
& +\sum_{j=0}^{N-1}\left\{\frac{l_{K}}{4} \frac{M\left\|H_{A}^{j}\right\|^{2}}{\Delta t}+\frac{l_{K}}{2} \frac{\operatorname{Tr}\left(\left(I_{3}-F_{A}^{j}\right) J_{d}\right)}{\Delta t}\right\} \\
& -\sum_{K \in \mathcal{T}} \sum_{1 \leq j<N} \Delta t \mathbb{V}_{K}\left(\Lambda_{K}^{j}, \mathbf{x}_{K}^{j}\right) .
\end{aligned}
$$

\subsubsection{Lie group variational integrator}

The discrete evolution is obtained by applying discrete Hamilton's principle to the discrete action (42). Equivalently this consists in computing the discrete Euler-Lagrange equations for each node $a$. From (24), we get the following systems of discrete EulerLagrange equations

$$
\begin{aligned}
& T_{e}^{*} L_{\left(F_{a}^{j-1}, H_{a}^{j-1}\right)}\left(D_{F_{a}^{j-1}} \mathcal{L}_{a}^{j-1}, D_{H_{a}^{j-1}} \mathcal{L}_{a}^{j-1}\right) \\
& -\mathrm{Ad}_{\left(F_{a}^{j}, H_{a}^{j}\right)^{-1}} T_{e}^{*} L_{\left(F_{a}^{j}, H_{a}^{j}\right)}\left(D_{F_{a}^{j}} \mathcal{L}_{a}^{j}, D_{H_{a}^{j}} \mathcal{L}_{a}^{j}\right) \\
& +T_{e}^{*} L_{\left(\Lambda_{a}^{j}, \mathbf{x}_{a}^{j}\right)}\left(D_{\Lambda_{a}^{j}} \mathcal{L}_{a}^{j}, D_{\mathbf{x}_{a}^{j}} \mathcal{L}_{a}^{j}\right)=0,
\end{aligned}
$$

for all $a \in \mathcal{N}$, where $\mathcal{L}_{a}^{j}$ denotes the dependence of the discrete action $\mathfrak{S}_{d}$ on $\left(\Lambda_{a}^{j}, \mathbf{x}_{a}^{j}, F_{a}^{j}, H_{a}^{j}\right)$, similarly for $\mathcal{L}_{a}^{j-1}$. Recall that we denote by $\mathcal{N}$ the set of all nodes, by $\partial \mathcal{N}=\{0, A\}$ the set of boundary nodes, and by $\operatorname{int}(\mathcal{N})=\{1, \ldots, A-1\}$ the set of internal nodes. The equations are slightly different for $a \in \operatorname{int}(\mathcal{N})$ and $a \in \partial \mathcal{N}$. Indeed, for $a \in \operatorname{int}(\mathcal{N})$ the discrete Lagrangian $\mathcal{L}_{a}^{j}$ is

$$
\mathcal{L}_{a}^{j}=\frac{l_{K}}{2} \frac{M\left\|H_{a}^{j}\right\|^{2}}{\Delta t}+l_{K} \frac{\operatorname{Tr}\left(\left(I_{3}-F_{a}^{j}\right) J_{d}\right)}{\Delta t}-\sum_{K \ni a} \Delta t \mathbb{V}_{K}\left(\Lambda_{K}^{j}, \mathbf{x}_{K}^{j}\right),
$$

whereas, for a boundary node $a \in \partial \mathcal{N}$, it reads

$$
\mathcal{L}_{a}^{j}=\frac{l_{K}}{4} \frac{M\left\|H_{a}^{j}\right\|^{2}}{\Delta t}+\frac{l_{K}}{2} \frac{\operatorname{Tr}\left(\left(I_{3}-F_{a}^{j}\right) J_{d}\right)}{\Delta t}-\sum_{K \ni a} \Delta t \mathbb{V}_{K}\left(\Lambda_{K}^{j}, \mathbf{x}_{K}^{j}\right) .
$$


Note that in (44) the sum in the last term involves two spatial elements $K$, whereas in (45) the sum involves only one subinterval.

Remark 3 (Duality pairing) We recall that we identify the dual space $\mathfrak{s o}(3)^{*}$ with $\mathfrak{s o}(3)$ via the natural pairing of $\mathbb{R}^{3}$, i.e. $\langle\widehat{\mathbf{v}}, \widehat{\mathbf{w}}\rangle:=\mathbf{v} \cdot \mathbf{w}=\frac{1}{2} \operatorname{Tr}\left(\widehat{\mathbf{v}}^{T} \widehat{\mathbf{w}}\right)$, where $\widehat{\widehat{A}}: \mathbb{R}^{3} \rightarrow \mathfrak{s o}(3)$ is the hat map defined in (8). The tangent space at $\Lambda \in S O(3)$ reads $T_{\Lambda} S O(3)=$ $\{\Lambda \xi \mid \xi \in \mathfrak{s o}(3)\}$. We identify the cotangent space $T_{\Lambda}^{*} S O(3)$ with $T_{\Lambda} S O$ (3) using the left-invariant pairing $\left\langle\alpha_{\Lambda}, V_{\Lambda}\right\rangle=\frac{1}{2} \operatorname{Tr}\left(\alpha_{\Lambda}^{T} V_{\Lambda}\right)$. With this identification, the cotangent lift of left translation reads

$$
(\Lambda, \phi)^{-1}\left(\alpha_{\Lambda},(\phi, \mathbf{v})\right)=T^{*} L_{(\Lambda, \phi)}\left(\alpha_{\Lambda},(\phi, \mathbf{v})\right)=\left(\Lambda^{T} \alpha_{\Lambda}, \Lambda^{T} \mathbf{v}\right) \in \mathfrak{s e}(3) .
$$

Discrete Euler-Lagrange equations. For any matrix $M$, let $M^{(A)}:=\left(M-M^{T}\right) / 2$ its antisymmetrization. Below we shall use the following identities:

$$
-\frac{1}{2} \operatorname{Tr}\left(M^{(A)} \widehat{\xi}\right)=\left(M^{(A)}\right)^{\vee} \cdot \xi \text { and }\left(\left(\mathbf{v w}^{T}\right)^{(A)}\right)^{\vee}=\frac{1}{2} \mathbf{w} \times \mathbf{v}
$$

for all $\xi, \mathbf{v}, \mathbf{w} \in \mathbb{R}^{3}$ and $3 \times 3$ matrices $M$.

A long, but straightforward computation, using (18), the discrete action sum (42), and the discrete Euler-Lagrange equations (43), yields the following equations:

(A) Discrete Euler-Lagrange equations for rotations:

(i) Interior nodes $a \notin\{0, A\}$

$$
\frac{l_{K}}{\Delta t}\left(J_{d} F_{a}^{j-1}-\left(F_{a}^{j-1}\right)^{T} J_{d}\right)^{\vee}-\frac{l_{K}}{\Delta t}\left(F_{a}^{j} J_{d}-J_{d}\left(F_{a}^{j}\right)^{T}\right)^{\vee}=\left.\Delta t \mathbf{U}_{a}\right|_{t=t^{j}}
$$

where at $t=t^{j}$ we have

$$
\begin{aligned}
\mathbf{U}_{a}:= & \left(\Lambda_{a}\right)^{-1} D_{\Lambda_{a}} \mathbb{V}_{K} \\
= & \frac{1}{2} \mathbf{C}_{1}\left(\Lambda_{a}^{T} \frac{\Delta \mathbf{x}_{a-1}}{l_{K}}-\mathbf{E}_{3}\right) \times \Lambda_{a}^{T} \Delta \mathbf{x}_{a-1}+\frac{1}{2} \mathbf{C}_{1}\left(\Lambda_{a}^{T} \frac{\Delta \mathbf{x}_{a}}{l_{K}}-\mathbf{E}_{3}\right) \\
& \times \Lambda_{a}^{T} \Delta \mathbf{x}_{a}+\frac{1}{l_{K}}\left(\left(\left(I+\Lambda_{a+1}^{T} \Lambda_{a}\right)^{-1} \widehat{\mathbf{C}_{2} \psi_{a}}\left(\widehat{\psi}_{a}-2 I\right)\right)^{(A)}\right)^{\vee} \\
& +\frac{1}{l_{K}}\left(\left(\left(\Lambda_{a-1}^{T} \Lambda_{a}+I\right)^{-1} \widehat{\mathbf{C}_{2} \psi_{a-1}}\left(2 I-\widehat{\psi}_{a-1}\right) \Lambda_{a-1}^{T} \Lambda_{a}\right)^{(A)}\right)^{\vee} .
\end{aligned}
$$

(ii) Left node $a=0$

$$
\frac{l_{K}}{2 \Delta t}\left(J_{d} F_{0}^{j-1}-\left(F_{0}^{j-1}\right)^{T} J_{d}\right)^{\vee}-\frac{l_{K}}{2 \Delta t}\left(F_{0}^{j} J_{d}-J_{d}\left(F_{0}^{j}\right)^{T}\right)^{\vee}=\left.\Delta t \mathbf{U}_{0}\right|_{t=t^{j}}
$$


where at $t=t^{j}$ we have

$$
\begin{aligned}
\mathbf{U}_{0}:= & \left(\Lambda_{0}\right)^{-1} D_{\Lambda_{0}} \mathbb{V}_{K}=\frac{1}{2} \mathbf{C}_{1}\left(\Lambda_{0}^{T} \frac{\Delta \mathbf{x}_{0}}{l_{K}}-\mathbf{E}_{3}\right) \times \Lambda_{0}^{T} \Delta \mathbf{x}_{0} \\
& +\frac{1}{l_{K}}\left(\left(\left(I+\Lambda_{1}^{T} \Lambda_{0}\right)^{-1} \widehat{\mathbf{C}_{2} \psi_{0}}\left(\widehat{\psi}_{0}-2 I\right)\right)^{(A)}\right)^{\vee}
\end{aligned}
$$

(iii) Right node $a=A$

$$
\frac{l_{K}}{2 \Delta t}\left(J_{d} F_{A}^{j-1}-\left(F_{A}^{j-1}\right)^{T} J_{d}\right)^{\vee}-\frac{l_{K}}{2 \Delta t}\left(F_{A}^{j} J_{d}-J_{d}\left(F_{A}^{j}\right)^{T}\right)^{\vee}=\left.\Delta t \mathbf{U}_{A}\right|_{t=t^{j}}
$$

where at $t=t^{j}$ we have

$$
\begin{aligned}
\mathbf{U}_{A}:= & \left(\Lambda_{A}\right)^{-1} D_{\Lambda_{A}} \mathbb{V}_{K}=\frac{1}{2}\left[\mathbf{C}_{1}\left(\Lambda_{A}^{T} \frac{\Delta \mathbf{x}_{A-1}}{l_{K}}-\mathbf{E}_{3}\right) \times \Lambda_{A}^{T} \Delta \mathbf{x}_{A-1}\right] \\
& +\frac{1}{l_{K}}\left(\left(\left(\Lambda_{A-1}^{T} \Lambda_{A}+I\right)^{-1} \widehat{\mathbf{C}_{2} \psi_{A-1}}\left(2 I-\widehat{\psi}_{A-1}\right) \Lambda_{A-1}^{T} \Lambda_{A}\right)^{(A)}\right)^{\vee}
\end{aligned}
$$

(B) Discrete Euler-Lagrange equations for positions:

(i) Interior nodes $a \notin\{0, A\}$

$$
\frac{l_{K} M}{\Delta t} \Delta \mathbf{x}_{a}^{j}-\frac{l_{K} M}{\Delta t} \Delta \mathbf{x}_{a}^{j-1}=-\left.\Delta t \Lambda_{a} \mathbf{V}_{a}\right|_{t=t^{j}}
$$

where at $t=t^{j}$ we have

$$
\begin{aligned}
\Lambda_{a} \mathbf{V}_{a}:= & D_{\mathbf{x}_{a}} \mathbb{V}_{K} \\
= & \frac{1}{2}\left(\Lambda_{a-1}\right) \mathbf{C}_{1}\left(\Lambda_{a-1}^{T} \frac{\Delta \mathbf{x}_{a-1}}{l_{K}}-\mathbf{E}_{3}\right)+\frac{1}{2}\left(-\Lambda_{a}\right) \mathbf{C}_{1}\left(\Lambda_{a}^{T} \frac{\Delta \mathbf{x}_{a}}{l_{K}}-\mathbf{E}_{3}\right) \\
& +\frac{1}{2}\left(\Lambda_{a}\right) \mathbf{C}_{1}\left(\Lambda_{a}^{T} \frac{\Delta \mathbf{x}_{a-1}}{l_{K}}-\mathbf{E}_{3}\right)+\frac{1}{2}\left(-\Lambda_{a+1}\right) \mathbf{C}_{1}\left(\Lambda_{a+1}^{T} \frac{\Delta \mathbf{x}_{a}}{l_{K}}-\mathbf{E}_{3}\right) \\
& +l_{K} \mathbf{q} .
\end{aligned}
$$

(ii) Left node $a=0$

$$
\frac{l_{K} M}{2 \Delta t} \Delta \mathbf{x}_{0}^{j}-\frac{l_{K} M}{2 \Delta t} \Delta \mathbf{x}_{0}^{j-1}=-\left.\Delta t \Lambda_{0} \mathbf{V}_{0}\right|_{t=t^{j}}
$$


where, at $t=t^{j}$ we have

$$
\begin{aligned}
\Lambda_{0} \mathbf{V}_{0}:= & D_{\mathbf{x}_{0}} \mathbb{V}_{K}=\frac{1}{2}\left(-\Lambda_{0}\right) \mathbf{C}_{1}\left(\Lambda_{0}^{T} \frac{\Delta \mathbf{x}_{0}}{l_{K}}-\mathbf{E}_{3}\right) \\
& +\frac{1}{2}\left(-\Lambda_{1}\right) \mathbf{C}_{1}\left(\Lambda_{1}^{T} \frac{\Delta \mathbf{x}_{0}}{l_{K}}-\mathbf{E}_{3}\right)+\frac{l_{K}}{2} \mathbf{q} .
\end{aligned}
$$

(iii) Right node $a=A$

$$
\begin{aligned}
& \frac{l_{K} M}{2 \Delta t} \Delta \mathbf{x}_{A}^{j}-\frac{l_{K} M}{2 \Delta t} \Delta \mathbf{x}_{A}^{j-1}=-\left.\Delta t \Lambda_{A} \mathbf{V}_{A}\right|_{t=t^{j}} \\
& \Lambda_{A} \mathbf{V}_{A}:=D_{\mathbf{x}_{A}^{j}} \mathbb{V}_{K}=\frac{1}{2}\left(\Lambda_{A-1}\right) \mathbf{C}_{1}\left(\Lambda_{A-1}^{T} \frac{\Delta \mathbf{x}_{A-1}}{l_{K}}-\mathbf{E}_{3}\right) \\
& +\frac{1}{2}\left(\Lambda_{A}\right) \mathbf{C}_{1}\left(\Lambda_{A}^{T} \frac{\Delta \mathbf{x}_{A-1}}{l_{K}}-\mathbf{E}_{3}\right)+\frac{l_{K}}{2} \mathbf{q}
\end{aligned}
$$

Note that the equations for translation and rotation are coupled via the known quantities at past time nodes and hence they can be solved for the unknown configuration in a fully decoupled way. The later equations can be solved explicitly for the unknown translation while an iteration is necessary to solve for the unknown rotation (see Sect. 4 on numerical examples for further details).

Remark 4 (Discrete versus continuous) We compare the discrete equations of motion to the continuous equations (14). Given $F_{a}^{j}=\left(\Lambda_{a}^{j}\right)^{T} \Lambda_{a}^{j+1}$ as a relative rotation of cross-section associated to node $a$ between times $t^{j}$ and $t^{j+1}$ we note that the first line of equations (48)-(52) divided by the time step $\Delta t$ is the discrete analog of the term $J \dot{\omega}+\omega \times J \omega$ of (14). This is consistent with the analog term arising in the discrete Euler-Lagrange equations for rigid bodies obtained by Lie group variational integrators, see [36]. The right hand side of (48)-(52) corresponds to the contribution of the discrete potential force at time $t^{j}$. By comparing with (14), we observe that the right hand side is the discrete analog of the potential term in (14). The same holds for a comparison of (54)-(58) to the second equation in (14).

\subsubsection{Discrete body momenta and Legendre transforms}

In the case of the beam, the discrete momenta are $\left(\pi^{j}\right)^{ \pm}=\left(\left(\pi_{0}^{j}\right)^{ \pm}, \ldots,\left(\pi_{A}^{j}\right)^{ \pm}\right)$, where $\left(\pi_{a}^{j}\right)^{ \pm}$are the discrete body momenta corresponding to the node $a \in \mathcal{N}$. Each of these momenta read $\left(\pi_{a}^{j}\right)^{ \pm}=\left(\left(\Pi_{a}^{j}\right)^{ \pm},\left(\Gamma_{a}^{j}\right)^{ \pm}\right)$, where $\left(\Pi_{a}^{j}\right)^{ \pm}$are the discrete body angular momenta and $\left(\Gamma_{a}^{j}\right)^{ \pm}$are the discrete linear body momenta. From (27) we know that these discrete momenta are given by

$$
\begin{aligned}
\left(\pi_{a}^{j}\right)^{-}= & \left(\left(\Pi_{a}^{j}\right)^{-},\left(\Gamma_{a}^{j}\right)^{-}\right)=-T_{e}^{*} L_{\left(\Lambda_{a}^{j}, \mathbf{x}_{a}^{j}\right)}\left(D_{\Lambda_{a}^{j}} \mathcal{L}_{a}^{j}, D_{\mathbf{x}_{a}^{j}} \mathcal{L}_{a}^{j}\right) \\
& +\operatorname{Ad}_{\left(F_{a}^{j}, H_{a}^{j}\right)^{-1}} T_{e}^{*} L_{\left(F_{a}^{j}, H_{a}^{j}\right)}\left(D_{F_{a}^{j}} \mathcal{L}_{a}^{j}, D_{H_{a}^{j}} \mathcal{L}_{a}^{j}\right)
\end{aligned}
$$




$$
\begin{aligned}
\left(\pi_{a}^{j}\right)^{+} & =\left(\left(\Pi_{a}^{j}\right)^{+},\left(\Gamma_{a}^{j}\right)^{+}\right) \\
& =T_{e}^{*} L_{\left(F_{a}^{j-1}, H_{a}^{j-1}\right)}\left(D_{F_{a}^{j-1}} \mathcal{L}_{a}^{j-1}, D_{H_{a}^{j-1}} \mathcal{L}_{a}^{j-1}\right)
\end{aligned}
$$

Their concrete expression is easily obtained from the computations in Sect. 3.5.2. In particular, as we already mentioned, there are some slight differences between the formulas for interior nodes and for boundary nodes.

The discrete Euler-Lagrange equations (48)-(52) can be equivalently written as

$$
\left(\Pi_{a}^{j-1}\right)^{+}=\left(\Pi_{a}^{j}\right)^{-}, \quad a \in \mathcal{N}
$$

while the discrete Euler-Lagrange equations (54)-(58) can be equivalently written as

$$
\left(\Gamma_{a}^{j-1}\right)^{+}=\left(\Gamma_{a}^{j}\right)^{-}, \quad a \in \mathcal{N} .
$$

Recall from (26) that the expressions of the momenta appear in the discrete Legendre transforms, whose $a$-component read

$$
\begin{aligned}
& \left(\mathbb{F}^{-} \mathcal{L}_{d}^{j}\right)_{a}=\left(\left(\Lambda_{a}^{j}, \mathbf{x}_{a}^{j}\right),\left(\left(\Pi_{a}^{j}\right)^{-},\left(\Gamma_{a}^{j}\right)^{-}\right)\right) \\
& \left(\mathbb{F}^{+} \mathcal{L}_{d}^{j}\right)_{a}=\left(\left(\Lambda_{a}^{j}, \mathbf{x}_{a}^{j}\right)\left(F_{a}^{j}, H_{a}^{j}\right),\left(\left(\Pi_{a}^{j}\right)^{+},\left(\Gamma_{a}^{j}\right)^{+}\right)\right) .
\end{aligned}
$$

\subsubsection{Invariance and discrete momentum maps}

From the expression (41) of the discrete Lagrangian of the beam, we obtain that it is $H$-invariant if and only if the potential $\mathbb{V}_{K}$ is $H$-invariant. From (18), we see that when the conservative force $\mathbf{q}$ is absent, the Lagrangian is left- $S E$ (3)-invariant under the action $\Phi$ given by

$$
\Phi_{(A, \mathbf{v})}\left(\left(\Lambda_{a}^{j}, \mathbf{x}_{a}^{j}\right)_{a \in \mathcal{N}}\right)=\left(A \Lambda_{a}^{j}, \mathbf{v}+A \mathbf{x}_{a}^{j}\right)_{a \in \mathcal{N}}
$$

Note that this action is the left translation by the subgroup $S E(3) \subset S E(3)^{N}$ (diagonal inclusion). The Lie algebra inclusion $i: \mathfrak{s e}(3) \rightarrow \mathfrak{s e}(3)^{N}$ reads $i(\Omega, \mathbf{v})=(\Omega, \mathbf{v})_{a \in \mathcal{N}}$, and its dual map is given by

$$
i^{*}\left(\left(\Pi_{a}, \Gamma_{a}\right)_{a \in \mathcal{N}}\right)=\sum_{a \in \mathcal{N}}\left(\Pi_{a}, \Gamma_{a}\right) \in \mathfrak{s e}(3)^{*} .
$$

Using the general formula (28) relating the discrete momentum maps for left translation by subgroups and the discrete body momenta, together with the formula (31) for the coadjoint action for $S E(3)$, we get

$$
\begin{gathered}
\mathbf{J}_{\mathcal{L}_{d}}^{+}\left(\left(\Lambda^{j}, \mathbf{x}^{j}\right),\left(F^{j}, H^{j}\right)\right)=i^{*}\left(\left(\operatorname{Ad}_{\left(\Lambda_{a}^{j+1}, \mathbf{x}_{a}^{j+1}\right)^{-1}}\left(\left(\Pi_{a}^{j}\right)^{+},\left(\Gamma_{a}^{j}\right)^{+}\right)\right)_{a \in \mathcal{N}}\right) \\
=\left(\sum_{a \in \mathcal{N}} \Lambda_{a}^{j+1}\left(\Pi_{a}^{j}\right)^{+}+\mathbf{x}_{a}^{j+1} \times \Lambda_{a}^{j+1}\left(\Gamma_{a}^{j}\right)^{+}, \sum_{a \in \mathcal{N}} \Lambda_{a}^{j+1}\left(\Gamma_{a}^{j}\right)^{+}\right) .
\end{gathered}
$$


Similarly, we get

$$
\mathbf{J}_{\mathcal{L}_{d}}^{-}\left(\left(\Lambda^{j}, \mathbf{x}^{j}\right),\left(F^{j}, H^{j}\right)\right)=\left(\sum_{a \in \mathcal{N}} \Lambda_{a}^{j}\left(\Pi_{a}^{j}\right)^{-}+\mathbf{x}_{a}^{j} \times \Lambda_{a}^{j}\left(\Gamma_{a}^{j}\right)^{-}, \sum_{a \in \mathcal{N}} \Lambda_{a}^{j}\left(\Gamma_{a}^{j}\right)^{-}\right) .
$$

By the general theory developed earlier, these momentum maps coincide since the discrete Lagrangian is $S E$ (3)-invariant.

The discrete Noether theorem (30) ensures that when the discrete Euler-Lagrange equations (48)-(58) are fulfilled, then $\mathbf{J}_{\mathcal{L}_{d}}$ is conserved in $\mathfrak{s e}(3)^{*}$, i.e.

$$
\mathbf{J}_{\mathcal{L}_{d}}\left(\left(\Lambda^{j}, \mathbf{x}^{j}\right),\left(F^{j}, H^{j}\right)\right)=\mathbf{J}_{\mathcal{L}_{d}}\left(\left(\Lambda^{j-1}, \mathbf{x}^{j-1}\right),\left(F^{j-1}, H^{j-1}\right)\right)
$$

We denote

$$
\left(\mathbf{J}_{\text {ang }}^{j}, \mathbf{J}_{\text {lin }}^{j}\right):=\mathbf{J}_{\mathcal{L}_{d}}\left(\left(\Lambda^{j}, \mathbf{x}^{j}\right),\left(F^{j}, H^{j}\right)\right)
$$

the discrete angular and linear momentum map.

In general, the presence of external forces breaks the $S E$ (3) symmetry. For example if we consider the gravity force $\mathbf{q}_{a}=-m_{a} g \mathbf{E}_{3}$, then the discrete Lagrangian is $S^{1}$ invariant under the $S^{1}$-action

$$
\Phi_{\theta}\left(\left(\Lambda_{a}^{j}, \mathbf{x}_{a}^{j}\right)_{a \in \mathcal{N}}\right)=\left(\exp \left(\theta \widehat{\mathbf{E}}_{3}\right) \Lambda_{a}^{j}, \exp \left(\theta \widehat{\mathbf{E}}_{3}\right) \mathbf{x}_{a}^{j}\right)_{a \in \mathcal{N}}
$$

In this case, the Lie algebra inclusion $i: \mathbb{R} \rightarrow \mathfrak{s e}(3)^{N}$ reads $i(\theta)=\left(\theta \widehat{\mathbf{E}}_{3}, 0\right)_{a \in \mathcal{N}}$, and its dual map is given by

$$
i^{*}\left(\left(\Pi_{a}, \Gamma_{a}\right)_{a \in \mathcal{N}}\right)=\mathbf{E}_{3} \cdot \sum_{a \in \mathcal{N}} \Pi_{a} \in \mathbb{R}
$$

Applying the same formulas as above, the discrete momentum maps are

$$
\begin{aligned}
& \mathbf{J}_{\mathcal{L}_{d}}^{+}\left(\left(\Lambda^{j}, \mathbf{x}^{j}\right),\left(F^{j}, H^{j}\right)\right)=\mathbf{E}_{3} \cdot \sum_{a \in \mathcal{N}} \Lambda_{a}^{j+1}\left(\Pi_{a}^{j}\right)^{+}+\mathbf{x}_{a}^{j+1} \times \Lambda_{a}^{j+1}\left(\Gamma_{a}^{j}\right)^{+} \\
& \mathbf{J}_{\mathcal{L}_{d}}^{-}\left(\left(\Lambda^{j}, \mathbf{x}^{j}\right),\left(F^{j}, H^{j}\right)\right)=\mathbf{E}_{3} \cdot \sum_{a \in \mathcal{N}} \Lambda_{a}^{j}\left(\Pi^{j}\right)_{a}^{-}+\mathbf{x}_{a}^{j} \times \Lambda_{a}^{j}\left(\Gamma_{a}^{j}\right)^{-} .
\end{aligned}
$$

As above, these two momentum maps coincide and the discrete Noether theorem ensures its conservation.

\subsubsection{Approximate energy conservation}

The spatially discretized energy is given by the Hamiltonian $\mathcal{H}$ associated to the Lagrangian (21) via Legendre transformation. We work with the trivialized expression of $\mathcal{H}$ given by 


$$
\begin{gathered}
\mathcal{H}\left(\left(\Lambda_{a}, \mathbf{x}_{a}, \Pi_{a}, \Gamma_{a}\right)_{a \in \mathcal{N}}\right)=\sum_{a \in \mathcal{N}}\left(\frac{1}{2 l_{K} M}\left\|\Gamma_{a}\right\|^{2}+\frac{1}{2 l_{K}}\left(J^{-1} \Pi_{a}\right)^{T} \Pi_{a}\right) \\
+\sum_{a \in \operatorname{int}(\mathcal{N})}\left(\frac{1}{l_{K} M}\left\|\Gamma_{a}\right\|^{2}+\frac{1}{l_{K}}\left(J^{-1} \Pi_{a}\right)^{T} \Pi_{a}\right)+\sum_{K \in \mathcal{T}} \mathbb{V}_{K}\left(\mathbf{x}_{K}, \Lambda_{K}\right) .
\end{gathered}
$$

\subsubsection{Initial conditions}

Suppose that the initial configuration of the continuous system on $G$ is given by $(g(0), \xi(0)) \in G \times \mathfrak{g}$. In order to solve the discrete Euler-Lagrange equations

$$
g^{j-1} f^{j-1}=g^{j} \text { and }\left(\pi^{j-1}\right)^{+}=\left(\pi^{j}\right)^{-},
$$

we have to initialize them by choosing $g^{0}$ and $f^{0}$. Given the initial conditions $(g(0), \xi(0))$ we define $g^{0}:=g(0),\left(\pi^{0}\right)^{+}=: \frac{\partial \mathcal{L}}{\partial \xi}(g(0), \xi(0))$, where $\mathcal{L}$ is the continuous Lagrangian, and $f^{0}$ is defined by solving the equation

$$
\left(f^{0}\right)^{-1} D_{f^{0}} \mathcal{L}_{d}\left(g^{0}, f^{0}\right)=\frac{\partial \mathcal{L}}{\partial \xi}(g(0), \xi(0)),
$$

where the left term of the equation is defined in (27).

\subsubsection{External torques and forces}

As we mentioned in Sect. 3.3.1, external forces can be incorporated in the variational integrator by using the discrete Lagrange-d'Alembert variational principle. A spatial discretization yields expressions $F\left(\left(\Lambda_{a}, \mathbf{x}_{a}, \dot{\Lambda}_{a}, \dot{\mathbf{x}}_{a}\right)_{a \in \mathcal{N}}\right)_{a}$ at each node. The time integral of the virtual work

$$
\int_{0}^{T} \sum_{a \in \mathcal{N}} F\left(\left(\Lambda_{a}, \mathbf{x}_{a}, \dot{\Lambda}_{a}, \dot{\mathbf{x}}_{a}\right)_{a \in \mathcal{N}}\right)_{a} \cdot\left(\delta \Lambda_{a}, \delta \mathbf{x}_{a}\right) d t
$$

done by these forces in the Lagrange-d'Alembert principle is then approximated via temporal discretization by an appropriate choice of the expressions $\mathcal{F}_{d}^{+}\left(\left(\Lambda^{j+1}, \mathbf{x}^{j+1}\right),\left(F^{j}, H^{j}\right)\right)_{a}$ and $\mathcal{F}_{d}^{-}\left(\left(\Lambda^{j}, \mathbf{x}^{j}\right),\left(F^{j}, H^{j}\right)\right)_{a}$. Here, for simplicity, we restrict to one-point quadrature by choosing $\mathcal{F}_{d}^{+}\left(\left(\Lambda^{j+1}, \mathbf{x}^{j+1}\right),\left(F^{j}, H^{j}\right)\right)_{a}=$ $(0,0)$ and

$$
\begin{aligned}
& \mathcal{F}_{d}^{-}\left(\left(\Lambda^{j}, \mathbf{x}^{j}\right),\left(F^{j}, H^{j}\right)\right)_{a} \\
& \quad=\Delta t\left(\mathfrak{M}_{a}^{-}\left(\left(\Lambda^{j}, \mathbf{x}^{j}\right),\left(F^{j}, H^{j}\right)\right), \mathfrak{F}_{a}^{-}\left(\left(\Lambda^{j}, \mathbf{x}^{j}\right),\left(F^{j}, H^{j}\right)\right)\right),
\end{aligned}
$$

an element of $T_{\left(\Lambda_{a}^{j}, \mathbf{x}_{a}^{j}\right)}^{*} S E(3)$. According to what was recalled in Sect. 3.3.1, in presence of external forces, the discrete body momenta $\left(\Pi_{a}^{j}\right)^{ \pm}$and $\left(\Gamma_{a}^{j}\right)^{ \pm}$are modified as follows 


$$
\left\{\begin{array}{l}
\left(\Pi_{\mathcal{F}, a}^{j}\right)^{+}=\left(\Pi_{a}^{j}\right)^{+} \\
\left(\Gamma_{\mathcal{F}, a}^{j}\right)^{+}=\left(\Gamma_{a}^{j}\right)^{+} \\
\left(\Pi_{\mathcal{F}, a}^{j}\right)^{-}=\left(\Pi_{a}^{j}\right)^{-}-\Delta t\left(\Lambda_{a}^{j}\right)^{-1} \mathfrak{M}_{a}^{-}\left(\left(\Lambda^{j}, \mathbf{x}^{j}\right),\left(F^{j}, H^{j}\right)\right) \\
\left(\Gamma_{\mathcal{F}, a}^{j}\right)^{-}=\left(\Gamma_{a}^{j}\right)^{-}-\Delta t\left(\Lambda_{a}^{j}\right)^{-1} \mathfrak{F}_{a}^{-}\left(\left(\Lambda^{j}, \mathbf{x}^{j}\right),\left(F^{j}, H^{j}\right)\right) .
\end{array}\right.
$$

The discrete Lagrange-d'Alembert principle yields the equations

$$
\left(\Pi_{\mathcal{F}, a}^{j-1}\right)^{+}=\left(\Pi_{\mathcal{F}, a}^{j}\right)^{-} \text {and }\left(\Gamma_{\mathcal{F}, a}^{j-1}\right)^{+}=\left(\Gamma_{\mathcal{F}, a}^{j}\right)^{-},
$$

or, using (62), by

$$
\left\{\begin{array}{c}
\left(\Pi_{a}^{j-1}\right)^{+}=\left(\Pi_{a}^{j}\right)^{-}-\Delta t\left(\Lambda_{a}^{j}\right)^{-1} \mathfrak{M}_{a}^{-}\left(\left(\Lambda^{j}, \mathbf{x}^{j}\right),\left(F^{j}, H^{j}\right)\right) \\
\left(\Gamma_{a}^{j-1}\right)^{+}=\left(\Gamma_{a}^{j}\right)^{-}-\Delta t\left(\Lambda_{a}^{j}\right)^{-1} \mathfrak{F}_{a}^{-}\left(\left(\Lambda^{j}, \mathbf{x}^{j}\right),\left(F^{j}, H^{j}\right)\right) .
\end{array}\right.
$$

In absence of external forces, one recovers the discrete Euler-Lagrange equations (48)-(58).

\subsection{Alternative variational integrator for the beam}

In this subsection we develop the variational integrator for the beam, by applying the alternative approach developed in Sect. 3.4.

\subsubsection{Time discretization}

The discrete Lagrangian $\mathscr{L}_{K}^{j}$, defined on $S E(3)^{N+1} \times \mathfrak{s e}(3)^{N+1}$, approximating the action of the Lagrangian $L_{K}$ in (20) over the interval $\left[t^{j}, t^{j+1}\right]$, for elements $K$ of length $l_{K}$, is

$$
\mathscr{L}_{K}^{j}=\Delta t \frac{l_{K}}{4} \sum_{a \in K}\left\{M\left\|\boldsymbol{\gamma}_{a}^{j}\right\|^{2}+\left(\omega_{a}^{j}\right)^{T} J \omega_{a}^{j}\right\}-\Delta t \mathbb{V}_{K}\left(\Lambda_{K}^{j}, \mathbf{x}_{K}^{j}\right),
$$

where, following (34), we define the discrete velocity $\xi_{a}^{j}:=\left(\omega_{a}^{j}, \boldsymbol{\gamma}_{a}^{j}\right) \in \mathfrak{s e}(3)$ in terms of the discrete Lie group elements $g_{a}^{j}=\left(\Lambda_{a}^{j}, \mathbf{x}_{a}^{j}\right)$, and $g_{a}^{j+1}=\left(\Lambda_{a}^{j+1}, \mathbf{x}_{a}^{j+1}\right) \in S E(3)$ by

$$
\begin{aligned}
\Delta t\left(\omega_{a}^{j}, \boldsymbol{\gamma}_{a}^{j}\right) & =\Delta t \xi_{a}^{j}=\tau^{-1}\left(\left(g_{a}^{j}\right)^{-1} g_{a}^{j+1}\right) \\
& =\tau^{-1}\left(\left(\Lambda_{a}^{j}\right)^{-1} \Lambda_{a}^{j+1},\left(\Lambda_{a}^{j}\right)^{-1} \Delta \mathbf{x}_{a}^{j}\right),
\end{aligned}
$$

where $\tau: \mathfrak{s e}(3) \rightarrow S E(3)$ is a local diffeomorphism in a neighborhood of the identity, with $\tau(e)=\mathbf{I}$. As in [32], we shall use the local diffeomorphism

$$
\tau(\omega, \boldsymbol{\gamma})=\left(\begin{array}{cc}
\operatorname{cay}(\omega) & \mathrm{d}^{R} \operatorname{cay}(\omega) \boldsymbol{\gamma} \\
0 & 1
\end{array}\right)
$$


where cay is the Cayley map of $S O(3)$, see (19). It is a local approximation of the exponential map of $S E(3)$, which is given in terms of the exponential map of $S O$ (3) by

$$
\exp (\omega, \boldsymbol{\gamma})=\left(\begin{array}{cc}
\exp (\omega) & \mathrm{d}^{R} \exp (\omega) \boldsymbol{\gamma} \\
0 & 1
\end{array}\right)
$$

Using (19), we can show that $\mathrm{d}^{R} \operatorname{cay}(\omega): \mathbb{R}^{3} \rightarrow \mathbb{R}^{3}$ is given by

$$
\mathrm{d}^{R} \operatorname{cay}(\omega) \boldsymbol{\gamma}=\frac{2}{4+\|\omega\|^{2}}\left(2 \mathbf{I}_{3}+\omega\right) \boldsymbol{\gamma}
$$

The matrix representation of the inverse of the right trivialized derivative $\mathrm{d}^{R} \tau^{-1}(\omega, \boldsymbol{\gamma})$ : $\mathbb{R}^{3} \times \mathbb{R}^{3} \rightarrow \mathbb{R}^{3} \times \mathbb{R}^{3}$ becomes

$$
\mathrm{d}^{R} \operatorname{cay}^{-1}(\omega, \boldsymbol{\gamma})=\left[\begin{array}{cc}
\mathbf{I}_{3}-\frac{1}{2} \omega+\frac{1}{4} \boldsymbol{\omega} \boldsymbol{\omega}^{T} & \mathbf{0}_{3} \\
-\frac{1}{2}\left(\mathbf{I}_{3}-\frac{1}{2} \omega\right) \gamma & \mathbf{I}_{3}-\frac{1}{2} \omega
\end{array}\right] .
$$

Since we are using the pairing $\langle(\boldsymbol{\mu}, \boldsymbol{\eta}),(\boldsymbol{\omega}, \boldsymbol{\gamma})\rangle=\boldsymbol{\mu} \cdot \boldsymbol{\omega}+\boldsymbol{\eta} \cdot \boldsymbol{\gamma}$ between $\mathfrak{s e}(3) \simeq \mathbb{R}^{3} \times \mathbb{R}^{3}$ and its dual $\mathfrak{s e}^{*}(3) \simeq \mathbb{R}^{3} \times \mathbb{R}^{3}$, the matrix of $\mathrm{d}^{R}$ cay $^{-1}(\omega, \boldsymbol{\gamma})^{*}$ is the transpose of (65).

One can also use for $\tau$ the Cayley map of $S E(3)$ given by

$$
\operatorname{cay}(\omega, \boldsymbol{\gamma})=\left(\begin{array}{cc}
\operatorname{cay}(\omega) & \left(I-\frac{\omega}{2}\right)^{-1} \boldsymbol{\gamma} \\
0 & 1
\end{array}\right)
$$

The discrete action, which approximates the continuous action over the time interval $[0, T]$, is therefore given by

$$
\begin{aligned}
\mathfrak{S}_{d}= & \sum_{K \in \mathcal{T}} \sum_{j=1}^{N-1} \mathscr{L}_{K}^{j}=\Delta t \frac{l_{K}}{2} \sum_{a \neq 0, A} \sum_{j=0}^{N-1}\left\langle\Delta \xi_{a}^{j}, \xi_{a}^{j}\right\rangle+\Delta t \frac{l_{K}}{4} \sum_{j=0}^{N-1}\left\langle d \xi_{0}^{j}, \xi_{0}^{j}\right\rangle \\
& +\Delta t \frac{l_{K}}{4} \sum_{j=0}^{N-1}\left\langle\mathbb{J} \xi_{A}^{j}, \xi_{A}^{j}\right\rangle-\Delta t \sum_{K \in \mathcal{T}} \sum_{j=1}^{N-1} \mathbb{V}_{K}\left(\Lambda_{K}^{j}, \mathbf{x}_{K}^{j}\right),
\end{aligned}
$$

where $\xi_{a}^{j}:=\left(\omega_{a}^{j}, \boldsymbol{\gamma}_{a}^{j}\right)$ and $\mathbb{J}: \mathbb{R}^{3} \times \mathbb{R}^{3} \rightarrow \mathbb{R}^{3} \times \mathbb{R}^{3}$ is the linear operator with matrix

$$
\mathbb{J}=\left(\begin{array}{cc}
J & 0 \\
0 & M \mathbf{I}_{3}
\end{array}\right)
$$

By evaluating the potential term $\mathbb{V}_{K}$ at time $t^{j}$, in the discrete Lagrangian (63), the dependence of the discrete action $\mathfrak{S}_{d}$ on $\left(\mathbf{x}_{a}^{j}, \Lambda_{a}^{j}, H_{a}^{j}, \Psi_{a}^{j}\right)$ reads 
(i) Interior nodes $a \notin\{0, A\}$

$$
\mathscr{L}_{a}^{j}=\Delta t \frac{l_{K}}{2}\left\langle\mathbb{J} \xi_{a}^{j}, \xi_{a}^{j}\right\rangle-\Delta t \sum_{K \ni a} \mathbb{V}_{K}\left(\Lambda_{K}^{j}, \mathbf{x}_{K}^{j}\right),
$$

(ii) Boundary nodes $a \in\{0, A\}$

$$
\mathscr{L}_{a}^{j}=\Delta t \frac{l_{K}}{4}\left\langle\rrbracket \xi_{a}^{j}, \xi_{a}^{j}\right\rangle-\Delta t \sum_{K \ni a} \mathbb{V}_{K}\left(\Lambda_{K}^{j}, \mathbf{x}_{K}^{j}\right)
$$

where in the potential term we choose the elements $K$ containing $a$.

\subsubsection{Alternative discrete Lagrange-d'Alembert equations}

Discrete forces. Given the discrete Lagrangian $\mathscr{L}_{a}$ as defined in (66) and (67), we choose the following discretization of the forces

$$
\begin{aligned}
& \mathcal{F}_{a, d}^{+}\left(\left(\Lambda_{a}^{j+1}, \mathbf{x}_{a}^{j+1}\right),\left(\omega^{j}, \gamma^{j}\right)\right)=(0,0) \\
& \mathcal{F}_{a, d}^{-}\left(\left(\Lambda_{a}^{j}, \mathbf{x}_{a}^{j}\right),\left(\omega^{j}, \gamma^{j}\right)\right) \\
& \quad=\Delta t\left(\mathfrak{M}_{a}^{-}\left(\left(\Lambda_{a}^{j}, \mathbf{x}_{a}^{j}\right),\left(\omega^{j}, \gamma^{j}\right)\right), \mathfrak{F}_{a}^{-}\left(\left(\Lambda_{a}^{j}, \mathbf{x}_{a}^{j}\right),\left(\omega^{j}, \gamma^{j}\right)\right)\right) \in T_{\left(\Lambda_{a}^{j}, \mathbf{x}_{a}^{j}\right)}^{*} S E(3),
\end{aligned}
$$

where $\mathfrak{M}_{a}$ and $\mathfrak{F}_{a}$ are respectively the exterior moment and force applied in node $a$. This choice is consistent with the approximation we made for the potential term.

We now give the discrete Lagrange-d'Alembert equations (35), for a given diffeomorphism $\tau: \mathfrak{s e}(3) \rightarrow S E(3)$ in a neighborhood of the origin such that $\tau(0)=e$.

Discrete Euler-Lagrange equation for the left boundary $a=0$. The equations read

$$
\left\{\begin{array}{l}
-\mu_{0}^{j}+\operatorname{Ad}_{\tau\left(\Delta t \xi_{0}^{j-1}\right)^{*}} \mu_{0}^{j-1}+\Delta t\left(\begin{array}{c}
\left(\Lambda_{0}^{j}\right)^{-1}\left(\mathfrak{M}_{0}^{j}\right)^{-} \\
\left(\Lambda_{0}^{j}\right)^{-1}\left(\mathfrak{F}_{0}^{j}\right)^{-}
\end{array}\right)=\left.\Delta t\left(\begin{array}{c}
\mathbf{U}_{0} \\
\mathbf{V}_{0}
\end{array}\right)\right|_{t=t^{j}}, \\
\mu_{0}^{j}=\frac{l_{K}}{2}\left(\mathrm{~d}^{R} \tau^{-1}\left(\Delta t \xi_{0}^{j}\right)\right)^{*}\left(\begin{array}{c}
J \omega_{0}^{j} \\
M \gamma_{0}^{j}
\end{array}\right), \quad g_{0}^{j+1}=g_{0}^{j} \tau\left(\Delta t \xi_{0}^{j}\right),
\end{array}\right.
$$

with $\mathbf{U}_{0}$ given by (51) and $\mathbf{V}_{0}$ by (57). Recall, $\psi_{0}=$ cay $^{-1}\left(\Lambda_{0}^{T} \Lambda_{1}\right)$ and $\Delta \mathbf{x}_{0}=\mathbf{x}_{1}-\mathbf{x}_{0}$.

The discrete body momenta are

$$
\pi_{B}^{+}\left(g_{0}^{j}, \xi_{0}^{j}\right)=\operatorname{Ad}_{\tau\left(\Delta t \xi_{0}^{j}\right)} \mu_{0}^{j} \text { and } \pi_{B}^{-}\left(g_{0}^{j}, \xi_{0}^{j}\right)=\Delta t\left(\begin{array}{l}
\mathbf{U}_{0} \\
\mathbf{V}_{0}
\end{array}\right)+\mu_{0}^{j}
$$


Discrete Euler-Lagrange equation for the right boundary $a=A$. The equations read

$$
\left\{\begin{array}{l}
-\mu_{A}^{j}+\operatorname{Ad}_{\tau\left(\Delta t \xi_{A}^{j-1}\right)^{*}} \mu_{A}^{j-1}+\Delta t\left(\begin{array}{c}
\left(\Lambda_{A}^{j}\right)^{-1}\left(\mathfrak{M}_{A}^{j}\right)^{-} \\
\left(\Lambda_{A}^{j}\right)^{-1}\left(\mathfrak{F}_{A}^{j}\right)^{-}
\end{array}\right)=\left.\Delta t\left(\begin{array}{c}
\mathbf{U}_{A} \\
\mathbf{V}_{A}
\end{array}\right)\right|_{t=t^{j}}, \\
\mu_{A}^{j}=\frac{l_{K}}{2}\left(\mathrm{~d}^{R} \tau^{-1}\left(\Delta t \xi_{A}^{j}\right)\right)^{*}\left(\begin{array}{c}
J \omega_{A}^{j} \\
M \gamma_{A}^{j}
\end{array}\right), \quad g_{A}^{j+1}=g_{A}^{j} \tau\left(\Delta t \xi_{A}^{j}\right),
\end{array}\right.
$$

with $\mathbf{U}_{A}$ given by (53) and $\mathbf{V}_{A}$ by (59). Recall, $\psi_{A-1}=\operatorname{cay}^{-1}\left(\Lambda_{A-1}^{T} \Lambda_{A}\right)$ and $\Delta \mathbf{x}_{A-1}=\mathbf{x}_{A}-\mathbf{x}_{A-1}$.

The discrete body momenta are

$$
\pi_{B}^{+}\left(g_{A}^{j}, \xi_{A}^{j}\right)=\operatorname{Ad}_{\tau\left(\Delta t \xi_{A}^{j}\right)} \mu_{A}^{j} \text { and } \pi_{B}^{-}\left(g_{A}^{j}, \xi_{A}^{j}\right)=\Delta t\left(\begin{array}{c}
\mathbf{U}_{A} \\
\mathbf{V}_{A}
\end{array}\right)+\mu_{A}^{j}
$$

Discrete Euler-Lagrange equation for a node a $\notin\{0, A\}$. The equations read

$$
\left\{\begin{array}{l}
-\mu_{a}^{j}+\operatorname{Ad}_{\tau\left(\Delta t \xi_{a}^{j-1}\right)}^{*} \mu_{a}^{j-1}+\Delta t\left(\begin{array}{c}
\left(\Lambda_{a}^{j}\right)^{-1}\left(\mathfrak{M}_{a}\right)^{-} \\
\left(\Lambda_{a}^{j}\right)^{-1}\left(\mathfrak{F}_{a}\right)^{-}
\end{array}\right)=\left.\Delta t\left(\begin{array}{c}
\mathbf{U}_{a} \\
\mathbf{V}_{a}
\end{array}\right)\right|_{t=t^{j}}, \\
\mu_{a}^{j}=l_{K}\left(\mathrm{~d}^{R} \tau^{-1}\left(\Delta t \xi_{a}^{j}\right)\right)^{*}\left(\begin{array}{c}
J \omega_{a}^{j} \\
M \boldsymbol{\gamma}_{a}^{j}
\end{array}\right), \quad g_{a}^{j+1}=g_{a}^{j} \tau\left(\Delta t \xi_{a}^{j}\right),
\end{array}\right.
$$

with $\mathbf{U}_{a}$ given by (49), $\mathbf{V}_{a}$ by (55), $\psi_{a}=\operatorname{cay}^{-1}\left(\Lambda_{a}^{T} \Lambda_{a+1}\right), \psi_{a-1}=\operatorname{cay}^{-1}\left(\Lambda_{a-1}^{T} \Lambda_{a}\right)$, $\Delta \mathbf{x}_{a-1}=\mathbf{x}_{a}-\mathbf{x}_{a-1}$, and $\Delta \mathbf{x}_{a-1}=\mathbf{x}_{a}-\mathbf{x}_{a-1}$.

The discrete body momenta are

$$
\pi_{B}^{+}\left(g_{a}^{j}, \xi_{a}^{j}\right)=\operatorname{Ad}_{\tau\left(\Delta t \xi_{a}^{j}\right)} \mu_{a}^{j}, \quad \pi_{B}^{-}\left(g_{a}^{j}, \xi_{a}^{j}\right)=(\Delta t)\left(\begin{array}{c}
\mathbf{U}_{a} \\
\mathbf{V}_{a}
\end{array}\right)+\mu_{a}^{j}
$$

Discrete momentum maps. We note that when $\mathbf{q}=\mathbf{0}$, then the discrete Lagrangian (63) is invariant under the left action of $S E(3)$. When $\mathbf{q} \neq \mathbf{0}$, say $\mathbf{q}=$ $q \mathbf{E}_{3}$, then the discrete Lagrangian is only invariant under the $S^{1}$-action given by $\Phi_{\theta} \cdot\left(\Lambda_{a}^{j}, \mathbf{x}_{a}^{j}\right):=\left(\exp \left(\theta \widehat{\mathbf{E}}_{3}\right) \Lambda_{a}^{j}, \exp \left(\theta \widehat{\mathbf{E}}_{3}\right) \mathbf{x}_{a}^{j}\right)$, where $\theta \in \mathbb{R}$. From the expression (36) we deduce that the discrete momentum maps associated to the $S E$ (3) action read

$$
\left\{\begin{array}{l}
J_{\mathscr{L}_{d}}^{f+}\left(\left(\Lambda^{j}, \mathbf{x}^{j}\right),\left(\omega^{j}, \boldsymbol{\gamma}^{j}\right)\right)=\sum_{a \in \mathcal{N}} \mathrm{Ad}_{\left(\Lambda_{a}^{j}, \mathbf{x}_{a}^{j}\right)^{-1}} \mu_{a}^{j} \\
J_{\mathscr{L}_{d}}^{f-}\left(\left(\Lambda^{j}, \mathbf{x}^{j}\right),\left(\omega^{j}, \boldsymbol{\gamma}^{j}\right)\right)=\left(\sum_{a \in \mathcal{N}} \mathrm{Ad}_{\left(\Lambda_{a}^{*}, \mathbf{x}_{a}^{j}\right)^{-1}} \mu_{a}^{j}\right. \\
\left.\quad-D_{\left(\Lambda_{a}^{j}, \mathbf{x}_{a}^{j}\right)} \mathscr{L}_{d}\left(\left(\Lambda^{j}, \mathbf{x}^{j}\right),\left(\omega^{j}, \boldsymbol{\gamma}^{j}\right)\right)\left(\Lambda_{a}^{j}, \mathbf{x}_{a}^{j}\right)^{-1}-\left(\mathfrak{M}_{a}^{j}, \mathfrak{F}_{a}^{j}\right)\left(\Lambda_{a}^{j}, \mathbf{x}_{a}^{j}\right)^{-1}\right) .
\end{array}\right.
$$

To obtain the discrete momentum maps associated to the $S^{1}$ action, one simply multiplies the above expression by the vector $\left(\mathbf{E}_{3}, \mathbf{0}\right)$. Note that the term 
$D_{\left(\Lambda_{a}^{j}, \mathbf{x}_{a}^{j}\right)} \mathscr{L}_{d}\left(\left(\Lambda^{j}, \mathbf{x}^{j}\right),\left(\omega^{j}, \boldsymbol{\gamma}^{j}\right)\right)\left(\Lambda_{a}^{j}, \mathbf{x}_{a}^{j}\right)^{-1}$ appearing in the discrete momentum map can be explicitly written as

$$
\begin{aligned}
D_{\left(\Lambda_{a}^{j}, \mathbf{x}_{a}^{j}\right)} \mathscr{L}_{d}\left(\left(\Lambda^{j}, \mathbf{x}^{j}\right),\left(\omega^{j}, \boldsymbol{\gamma}^{j}\right)\right)\left(\Lambda_{a}^{j}, \mathbf{x}_{a}^{j}\right)^{-1} & =-\operatorname{Ad}_{\left(\Lambda_{a}^{j}, \mathbf{x}_{a}^{j}\right)^{-1}}\left(\mathbf{U}_{a}^{j}, \mathbf{V}_{a}^{j}\right) \\
& =-\left(\Lambda_{a}^{j} \mathbf{U}_{a}^{j}+\mathbf{x}_{a}^{j} \times \Lambda_{a}^{j} \mathbf{V}_{a}^{j}, \Lambda_{a}^{j} \mathbf{V}_{a}^{j}\right)
\end{aligned}
$$

where the expressions of $\mathbf{U}_{a}, \mathbf{V}_{a}$ are given in (49), (51), (53), (55), (57), and (59). This formula holds for both boundary nodes and interior nodes.

As recalled in (38), these discrete momentum maps (either in the $S^{1}$ or $S E$ (3) symmetric settings) are preserved if the discrete forces are absent or are orthogonal to the symmetry group action.

\subsubsection{Approximate energy conservation}

The trivialized expression of the discretized energy is given by the Hamiltonian $\mathscr{H}$ associated to the Lagrangian (63) given, in terms of the convected discrete velocities $\omega_{a}, \gamma_{a}$, by

$$
\begin{aligned}
& \mathscr{H}\left(\left(\Lambda_{a}, \mathbf{x}_{a}, \omega_{a}, \gamma_{a}\right)_{a \in \mathcal{N}}\right)=\sum_{a \in \partial \mathcal{N}} \frac{l_{K}}{4}\left(M\left\|\boldsymbol{\gamma}_{a}\right\|^{2}+\left(\boldsymbol{\omega}_{a}\right)^{T} J \boldsymbol{\omega}_{a}\right) \\
&+\sum_{a \in \operatorname{int}(\mathcal{N})} \frac{l_{K}}{2}\left(M\left\|\boldsymbol{\gamma}_{a}\right\|^{2}+\left(\omega_{a}\right)^{T} J \omega_{a}\right)+\sum_{K \in \mathcal{T}} \mathbb{V}_{K}\left(\mathbf{x}_{K}, \Lambda_{K}\right)
\end{aligned}
$$

\subsubsection{Comparison with other methods}

Our scheme is obtained by applying a variational integrator to a semidiscretization of the configuration space and the Lagrangian of the beam. In this sense, it is similar with other approaches, such as, e.g. $[13,25,41]$. However, the specificity of our approach lies in the fact that both the space and time discretizations are realized in a "Lie group preserving way" which implies that the symmetries of the original problem are preserved by the discretization. This allows us to define the discrete notions of angular and linear momentum maps in a consistent geometric way [50] and to obtain their exact conservation. Another benefit of our approach is that, since it preserves a Lie group structure, the spatial discretization in (16) can be easily combined with any Lie group methods for the temporal discretization, such as the ones in $[10,26]$, including the Runge-Kutta-Munthe-Kaas methods. Regarding the spatial discretization, any interpolation $\Lambda_{h}(S), \phi_{h}(S)$ of $\left(\Lambda_{a}, \mathbf{x}_{a}\right)_{a \in \mathcal{N}}$ that preserves the objectivity and the $S E$ (3) left action, can be used without altering our results. It can then be followed by any of the temporal Lie group methods mentioned above. Our setting is therefore potentially useful to derive higher order schemes.

Approaches to dynamic beam simulation being inherently different from Lie group methods, are already mentioned in the introduction. In particular, [5,34,35] use redundant coordinates like quaternions or director triads. To describe the rotational degrees 
of freedom, they need to be subject to constraints, leading to differential algebraic equations of motion that are treated, e.g., with index reduction or projection methods. An advantage of our approach is that it does not need the introduction of any additional constraint ensuring that the scheme preserves the Lie algebra of rotations, since this property is naturally guaranteed through the use of Lie group variational integrators. Furthermore, different spatial discretizations, e.g., using a staggered grid in [34], or finite elements in [5], or using a spatially discrete Lagrangian (21) as in this work, yield different mass matrices which influence the numerical results. For example, for the variational case, the mass is lumped into the nodes resulting in a diagonal mass matrix, while the finite element semidiscretization of space yields a tridiagonal mass matrix. Since the goal of $[34,35]$ is different than the one pursued in this paper, the authors do not provide any information concerning the symplecticity of the algorithm, the conservation of the symmetries (momentum maps), and of the energy. They choose to discretize the ODE, whereas, in our approach, we discretize directly Hamilton's principle in a way that preserves the Lie group symmetries, which ensures the symplecticity as well as the preservation of momentum maps.

We would like to mention that it is also possible to carry out a spacetime covariant (as opposed to spatial followed by temporal) Lie group preserving discretization of beam dynamics. Such a variational discretization has been carried out in [16] following the multisymplectic discretization of [47]. This approach allows for symplecticity in both the space and time evolutions, as well as a discrete version of covariant Noether theorem (that implies the classical one). However, imposing both the covariance and the "Lie group preserving" properties allows for less freedom for the choice of the spatial and temporal discretizations, than in the setting developed in the present paper. Therefore, it appears that both approaches have their own advantages. Note that through the discrete field theory point of view, some specific developments were introduced, such as the asynchronous variational integrators [38] and the semi-discrete variational integrators [15].

\section{Numerical results}

In this section, we numerically analyze the derived variational integrators by means of two numerical examples. To this end, we implement three different integrators: first of all the Lie group variational integrator for the beam given by equations (48)-(59), secondly the alternative variational integrator for the beam given by equations (69)(71) and thirdly an energy-momentum preserving time stepping scheme with finite elements in space as described in [41].

\subsection{Implementation details}

\subsubsection{Lie group variational integrator for the beam}

For the implementation of the Lie group variational integrator, we have in equations (54)-(59) an explicit update to determine $\Delta \mathbf{x}_{a}^{j}$, while (48)-(53) are an implicit expression of the form 


$$
\left(F_{a}^{j} J_{d}-J_{d}\left(F_{a}^{j}\right)^{T}\right)=\widehat{\mathbf{a}} .
$$

In order to solve this equation for $F_{a}^{j} \in S O(3)$, (the vector a and the symmetric matrix $J_{d}$ being given), we use a Newton iteration based on the Cayley transformation as described in [37] (Sect. 3.3.8). In the following we denote the variational Lie group integrator by 'lgvi'.

\subsubsection{Alternative variational integrator for the beam}

The alternative integrator we proposed in Sects. 3.4 and 3.6 involves the choice of a local diffeomorphism in (34). Once this diffeomorphism is chosen, no further approximation is needed to derive the scheme, in contrast with the first integrator, which involves an approximation of the velocity (40). The implementation is based on (69), (70) and (71), each consisting of three equations. For a given group element $g_{a}^{j}$, a given Lie algebra element $\xi_{a}^{j-1}$ and given $\mu_{a}^{j-1}, \mu_{a}^{j}$ is determined by the first equation as an explicit update. A Newton iteration is performed to solve the second equation for $\xi_{a}^{j}$, and finally, the group element is updated to $g^{j+1}$ by the explicit update in the third equation. In the following we denote the alternative variational integrator for by 'lavi'.

\subsubsection{Energy-momentum preserving time stepping scheme with finite elements in space}

For a comparison, the same problem is simulated using a well established energymomentum preserving time stepping scheme with finite elements in space as described in [41]. For further energy-momentum conserving simulations of geometrically exact beam dynamics using a finite element space discretization see e.g. [55]. In [41], the kinematics are represented via a constrained configuration variable, discretized with linear finite elements in space and the time discretization of the resulting constrained Hamiltonian system is performed via a $G$-equivariant discrete derivative introduced by [22]. In the discrete setting, the discrete null space method with nodal reparametrization is applied which reduces the computational effort and improves the system's condition, see [42]. The resulting discrete trajectory is exactly energy and momentum conserving (both quantities conserve their initial values up to the tolerance employed in the Newton iteration, which is in contrast to the good energy behavior of variational schemes where finite energy oscillations are present), but it is not symplectic. Theoretical analysis shows that the scheme converges with second order in space and time. In the following we denote the energy-momentum preserving time stepping scheme with finite elements in space by 'feem'.

\subsection{Beam with concentrated masses}

As a first example we consider a geometrically exact beam with a concentrated mass $m$ at the middle node and concentrated masses $M$ at the boundary nodes and with a three-dimensional loading acting on the concentrated masses (as depicted in Fig. 2). 


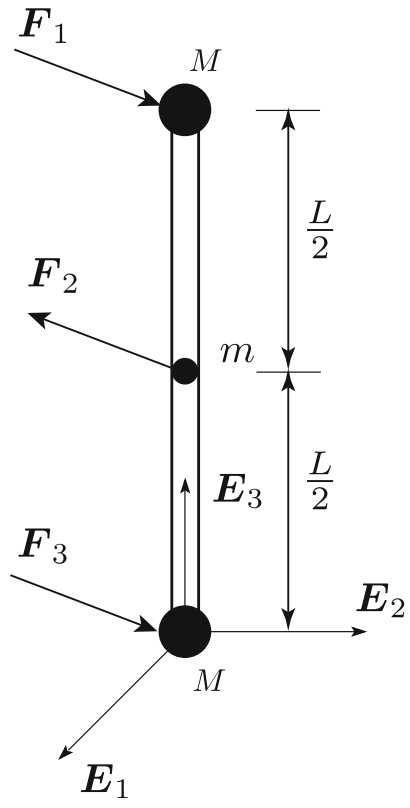

Fig. 2 Beam with concentrated masses

This is a standard benchmark example which has been previously addressed e.g. in [6] with slightly different loading. The beam is initially aligned along the $\mathbf{E}_{3}$ axis and undeformed. For this problem, the following parameters are used: beam length $L=2$, concentrated masses $M=10$ and $m=1$, mass density $\varrho=1,000$, square cross-section with edge length $a=0.05$, Poisson ratio $v=0.35$, and Young's modulus $E=10^{7}$. We consider this problem without external potential forces, such that $\Pi_{\text {ext }}(\phi)=0$.

The temporally bounded external loading has the form

$$
\mathbf{F}_{\kappa}(t)=f(t) \mathbf{P}_{k} \text { for } k=1,2,3
$$

with

$$
\begin{aligned}
\mathbf{P}_{1}=\mathbf{P}_{3} & =-1.0 \mathbf{E}_{1}+1.6 \mathbf{E}_{2}-1.2 \mathbf{E}_{3} \\
\mathbf{P}_{2} & =1.0 \mathbf{E}_{1}-1.6 \mathbf{E}_{2}+1.2 \mathbf{E}_{3}
\end{aligned}
$$

and the function

$$
f(t)= \begin{cases}100\left(1-\cos \left(\frac{2 \pi t}{T_{\text {load }}}\right)\right) & \text { for } t \leq T_{\text {load }} \\ 0 & \text { for } t>T_{\text {load }}\end{cases}
$$

for $T_{\text {load }}=0.1$. No other external loads are present in this example. Furthermore, the beam's initial translational velocity is linearly distributed as

$$
\dot{\phi}(S, 0)=\varphi(S) \mathbf{P}, \quad \mathbf{P}=\frac{1}{20}\left(1.0 \mathbf{E}_{1}+2.0 \mathbf{E}_{2}+3.0 \mathbf{E}_{3}\right)
$$


with the function

$$
\varphi(S)= \begin{cases}4-16 S / L & \text { for } S \leq L / 2 \\ -10+16 S / L & \text { for } S>L / 2\end{cases}
$$

and the initial rotational velocity $\Omega(S, 0)$ is zero.

For all three methods, two simulations are performed until the simulation time $T=0.9$. The first simulation is based on a constant time step $\Delta t=10^{-4}$ and an equidistant spatial discretization of the central line of the beam by $A=22$ beam elements which corresponds to an element length $\Delta l=l_{K}=\frac{L}{A}$ for all elements $K$. The second simulation uses the constant time step $\Delta t=10^{-5}$ and $A=66$ beam elements.

An impression of the motion and deformation of the spatially discretized beam is given in Fig. 3 by snapshots of consecutive configurations for a simulation using the variational Lie group integrator with $\Delta t=10^{-5}$ and $A=66$. The elements of the beam are colored by a linear interpolation of the sum of the norms of the stress resultants $\left\|\mathbf{n}_{K}\right\|+\left\|\mathbf{m}_{K}\right\|$ in the elements $K=1, \ldots, 66$, as defined in (22), (23).

For the discretization based on $\Delta t=10^{-5}$ and $A=66$, all three integrators provide similar beam motions. The similarity of the results can be observed Fig. 4 (right), which shows the time evolution of five selected nodes. However, for a more rough discretization with $\Delta t=10^{-4}$ and $A=22$, the numerical solution do not coincide anymore (see Fig. 4 (left)). Whereas lgvi and lavi show similar behavior, the solution of feem differs.

Also, the evolution of the energy and the stress resultants differ between the methods for simulations with $\Delta t=10^{-4}, A=22$. In Fig. 5, the energy ((61) for lgvi and (73) for lavi), the angular momentum and linear momentum ((60) for lgvi and (72) for lavi) of the beam are depicted for the three methods. The evolution of the stress resultants $\mathbf{n}_{K}$ (shear stresses and stretch) and $\mathbf{m}_{K}$ (bending moment and torsional moment) in the spatial elements 1, 12 and 22 is depicted in Fig. 6. It can be observed that lgvi and lavi compute a stronger deformation, in particular concerning the extension stress $\mathbf{n}_{K, 3}$. This yields a larger deformation energy compared to the feem, thus a smaller kinetic energy and a larger exchange of energy between the kinetic and the potential parts is predicted by the variational schemes.

All methods show a very good behavior concerning their structure preserving properties: as expected, from the discrete Noether theorem (Sect. 3.5.4), the two components (angular and linear) of the discrete momentum map $\mathbf{J}_{\mathcal{L}_{d}}$ associated with $S E$ (3)invariance are preserved up to numerical accuracy after the external loads vanish at $T_{\text {load }}=0.1$. Also the total energy of all schemes is conserved after this time. However, the value of the total energy differs between the variational schemes and feem due to the different space discretization, in particular, the mass matrix differs, see Sect. 3.6.4.

Energy, linear momentum, angular momentum, and stress resultants for the finer discretization ( $\Delta t=10^{-5}, A=66$ ) for all three methods are depicted in Figs. 7 and 8 , respectively. All three methods show similar behavior for this time and space discretization. Note that for the fine discretization, all methods show the same qualitative behavior which has been predicted by the feem already using the coarse discretization. 


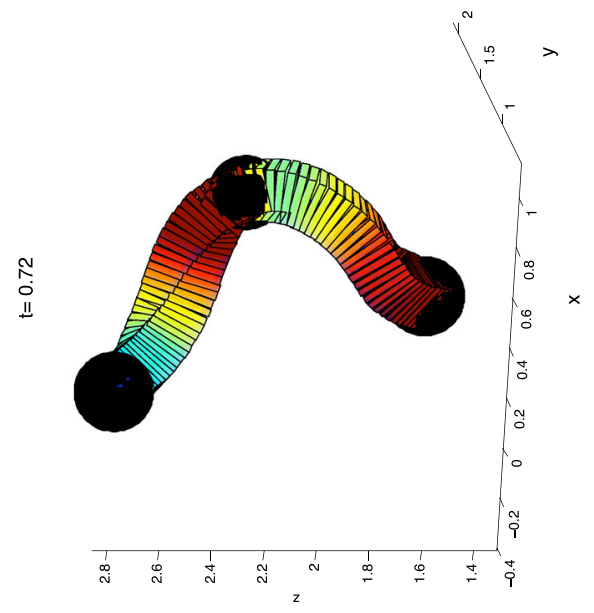

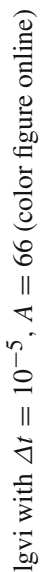

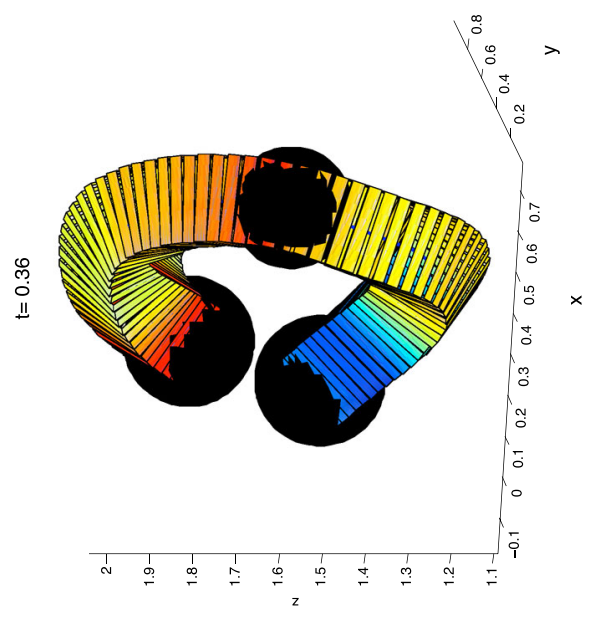

ำ

0
0
0

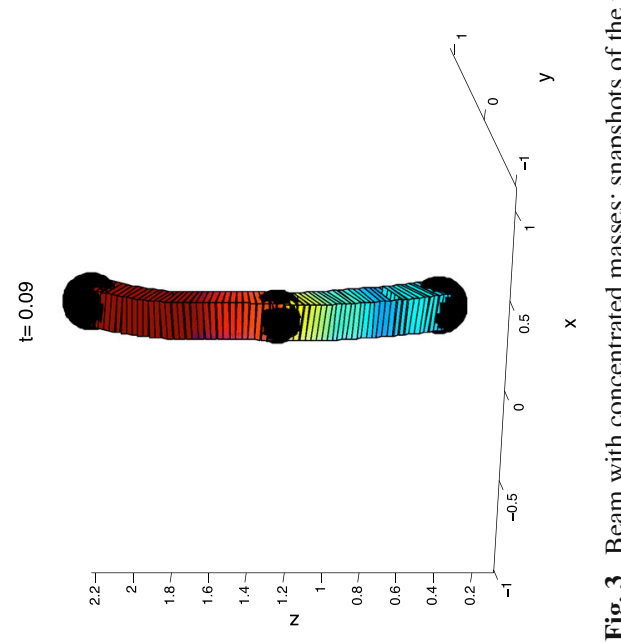




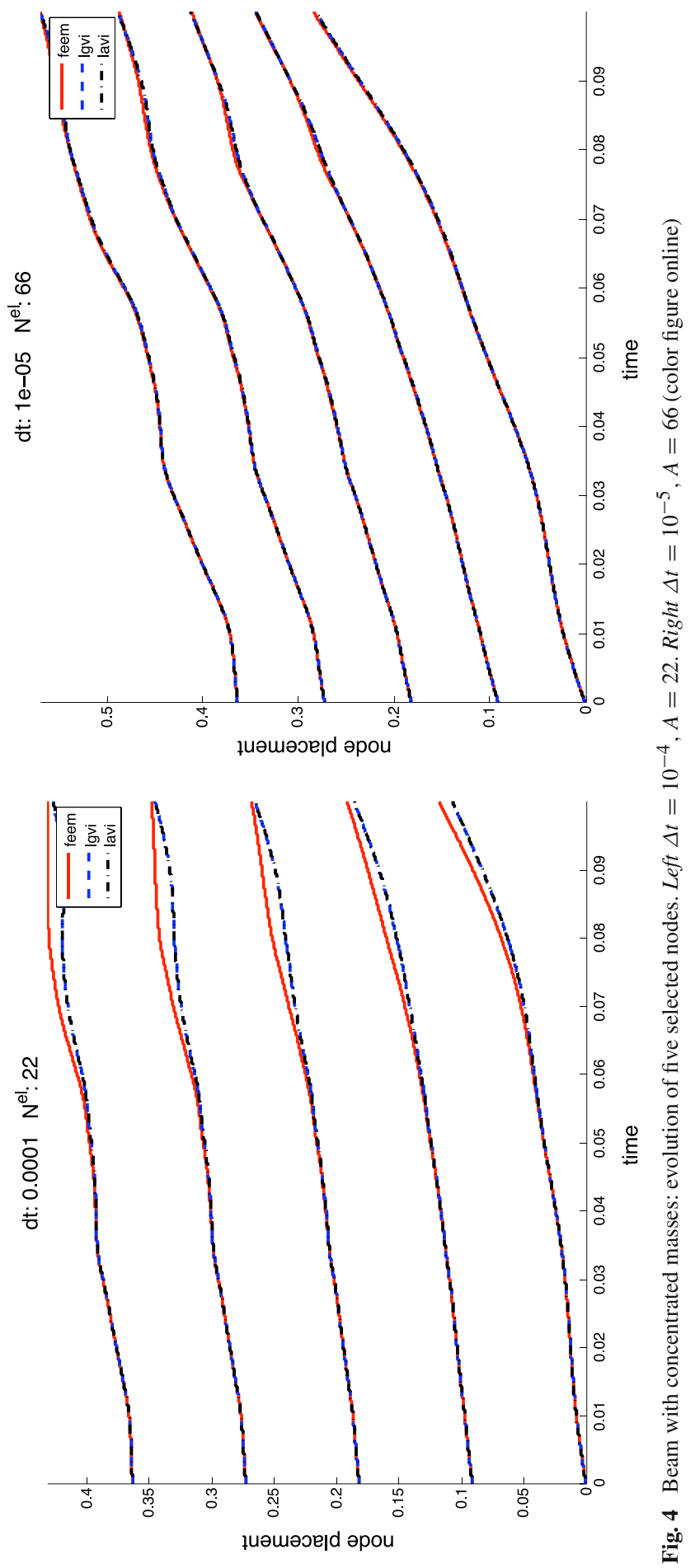




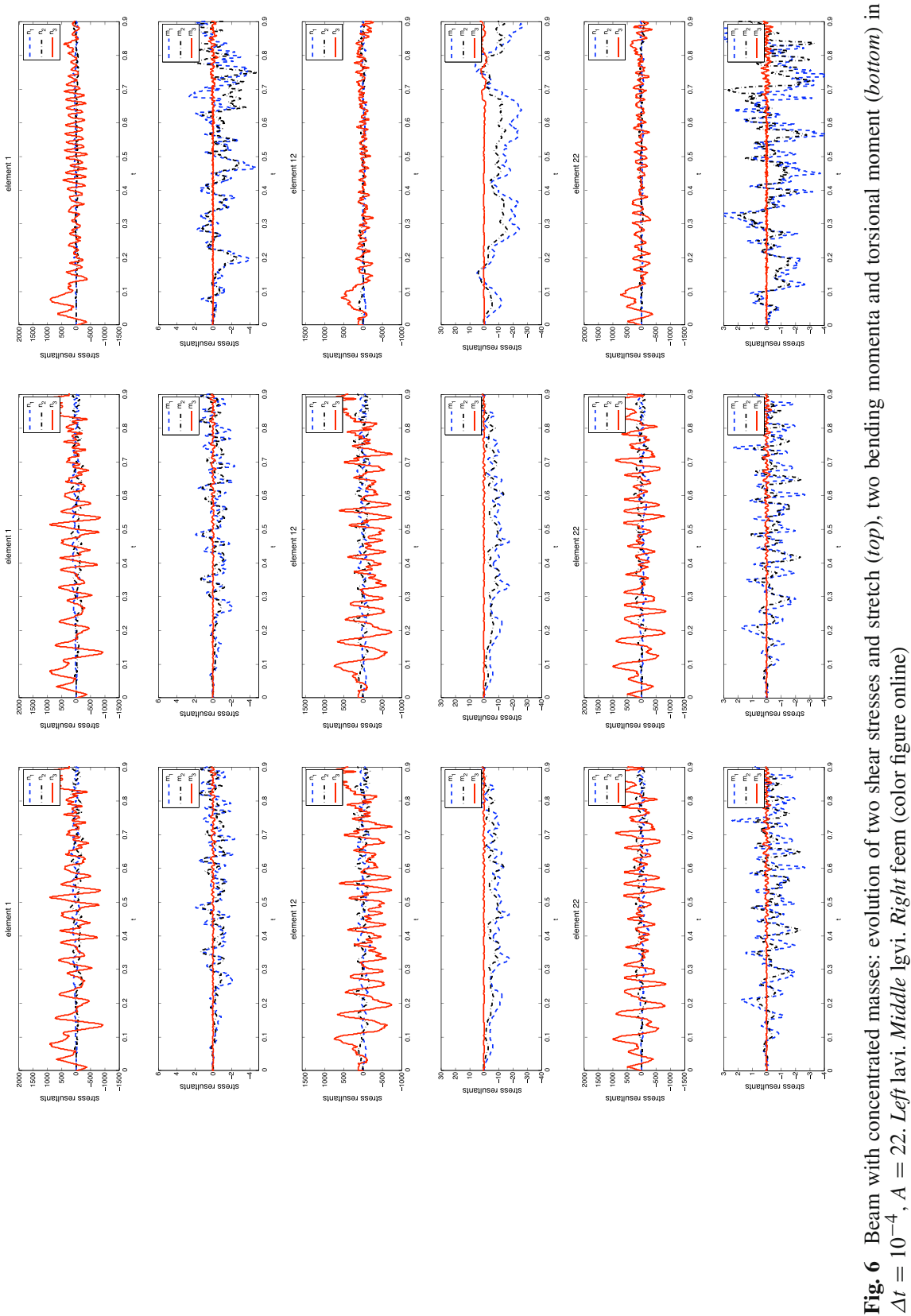


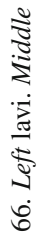
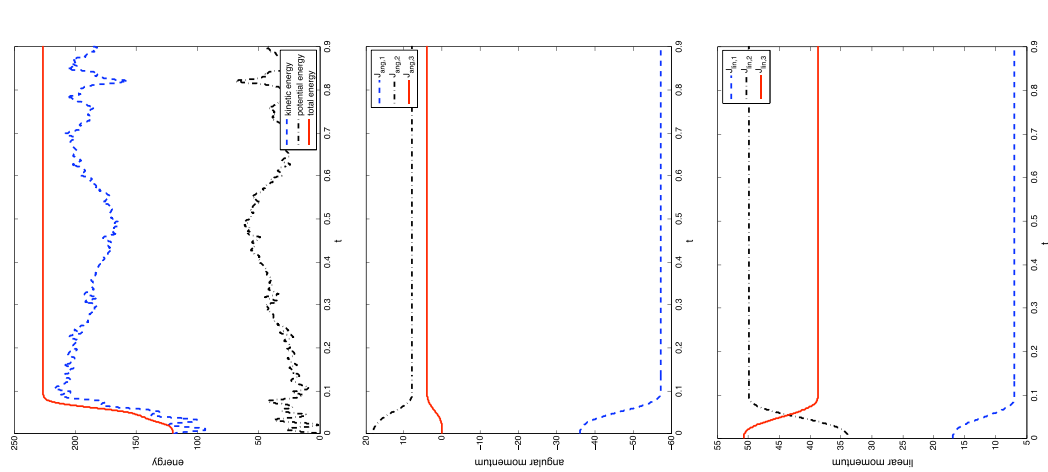

I

?
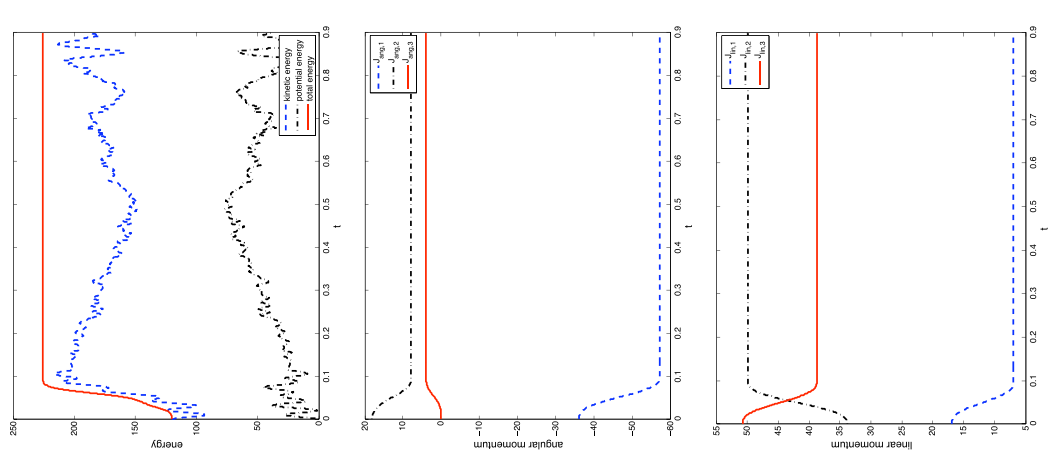

苛
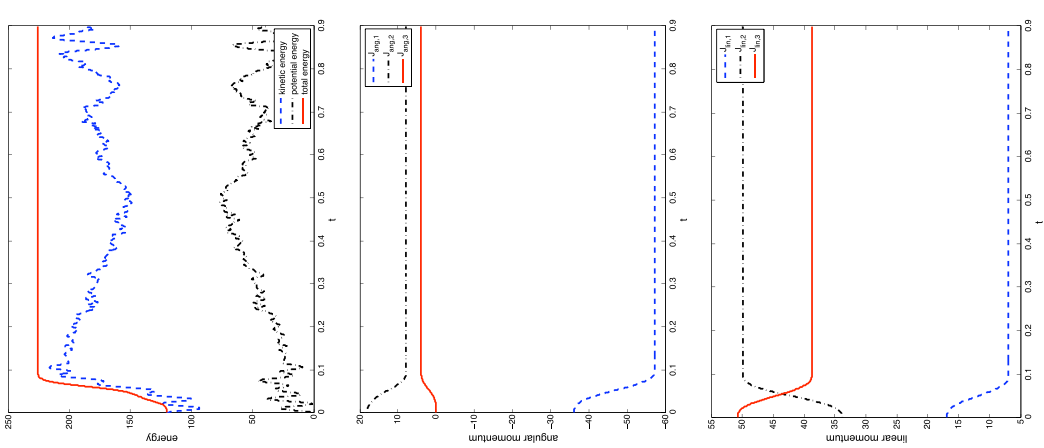

0
0
0
0
0
0
0
0
0
0
0
0
0
0
0
0
0

ن 

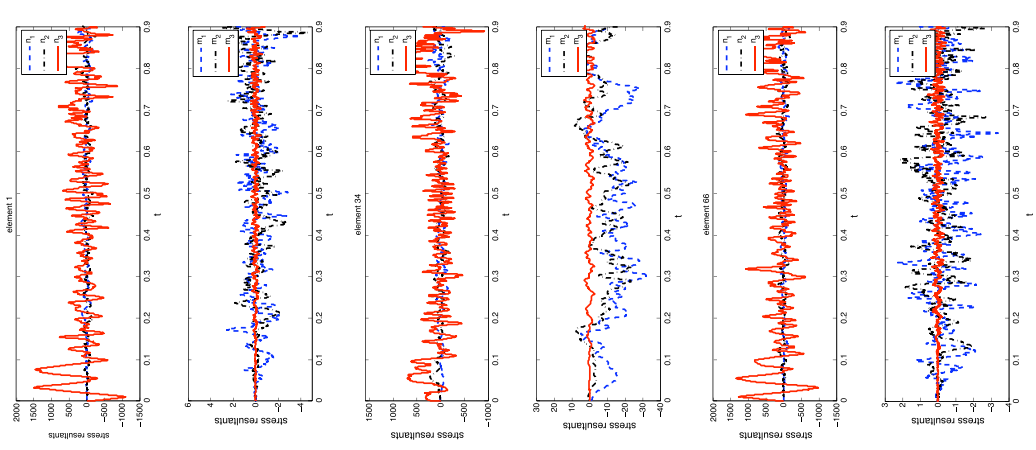

.

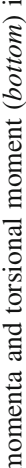
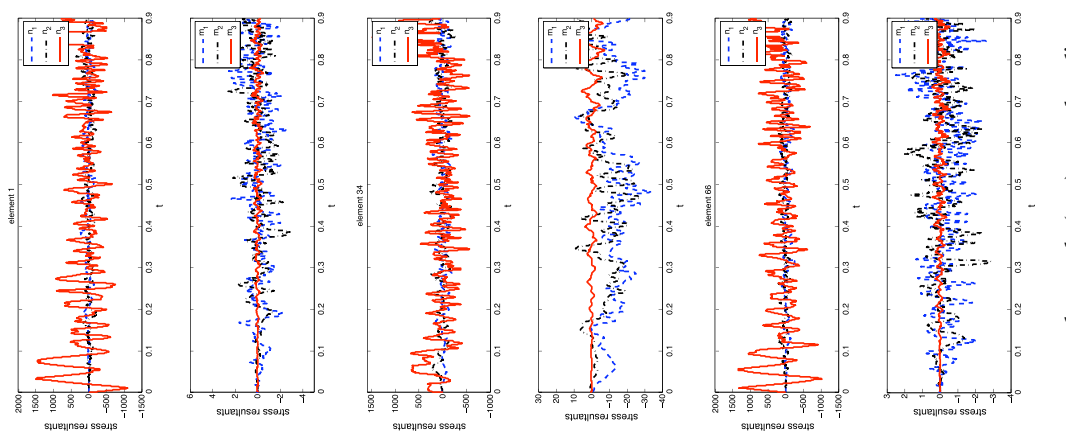

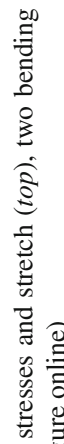
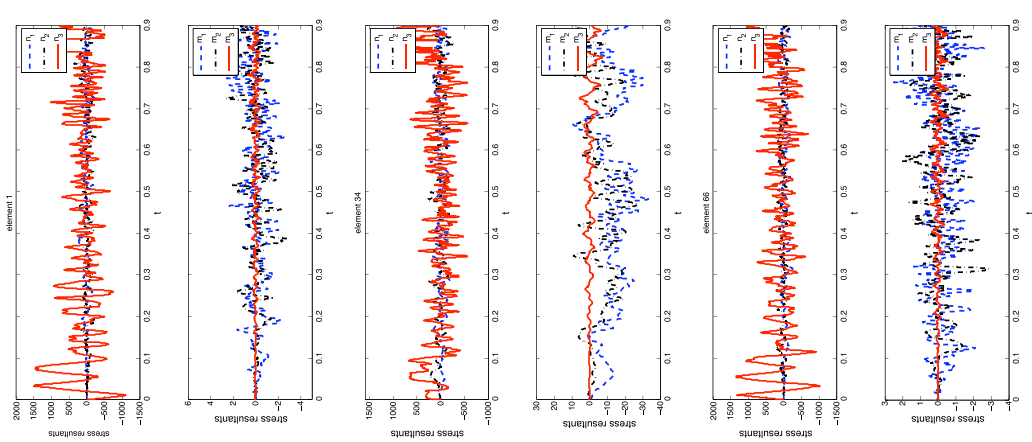

记

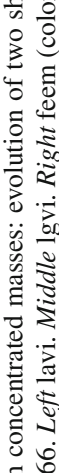

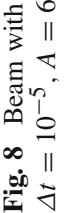


Thus, for a fine enough grid, all methods converge to the same numerical solution. This is confirmed by the following numerical convergence analysis.

All three methods show an excellent long term energy behavior. Small oscillations are visible for lgvi and lavi, while feem yields exact energy conservation, see Fig. 9.

\subsection{Numerical convergence analysis}

For the convergence analysis of variational integrators, techniques based on a variational error analysis using the exact discrete Lagrangian are typically used, see e.g. [50] and [52]. In this work, we restrict ourselves to a numerical convergence analysis and we investigate the following simple example scenario: We consider a geometrically exact beam lying in the $\left(\mathbf{E}_{1}, \mathbf{E}_{3}\right)$-plane with an initial deformation given as follows: the beam describes a circular arc with opening angle $\frac{2 \pi}{3}$ and it is compressed to $75 \%$ of its original length $L$. The beam is free to move in space and has zero initial velocity. Again, an equidistant spatial discretization is used. We choose the following parameters: beam length $L=2$, mass density $\varrho=1,000$, square cross-section with edge length $a=0.05$, Poisson ratio $v=0.35$, and Young's modulus $E=10^{4}$. For all three methods we investigate the convergence behavior to identify convergence rates and similarities and differences in their numerical results.

The numerical convergence analysis concentrates on two aspects. First of all, each method is analyzed separately with regard to the convergence for time grid refinement at constant space resolution, the convergence of space grid refinement at constant time resolution and with regard to the convergence for the simultaneous refinement of the space time grid and convergence rates are deduced numerically. While the first two experiments illustrate the convergence of the variational time integration and the variational space integration for finite dimensional systems, i.e. for the ODE case, respectively, the latter illustrates the convergence of the variational space time integration for the PDE case. Secondly, the differences between the methods are analyzed showing that the solution of one method does converge to the solution of another method and deduces the rate. Finally, the different behavior concerning the computational efficiency of the three methods is illustrated.

\subsubsection{Convergence of each method}

For each method, we perform simulations with the following number of elements $A=16,32,64,128,256$ and time step $\Delta t=1.6 \cdot 10^{-4}, 8 \cdot 10^{-5}, 4 \cdot 10^{-5}, 2$. $10^{-5}, 10^{-5}$. The simulation time is $T=0.06$ in all comparisons. The relative error $\varepsilon_{\text {rel }}$ for a simulation result is computed by the discrete $L_{2}$-norm of the relative error between the actual simulated configuration $g$ and a given reference configuration $g$ ref. To this end, the nodal relative error of a configuration $g_{a}^{j}=\left(\Lambda_{a}^{j}, \mathbf{x}_{a}^{j}\right)$ at a time and space node is computed by the 2-norm, resulting in the matrix of relative error $\varepsilon_{\text {rel }}$ 


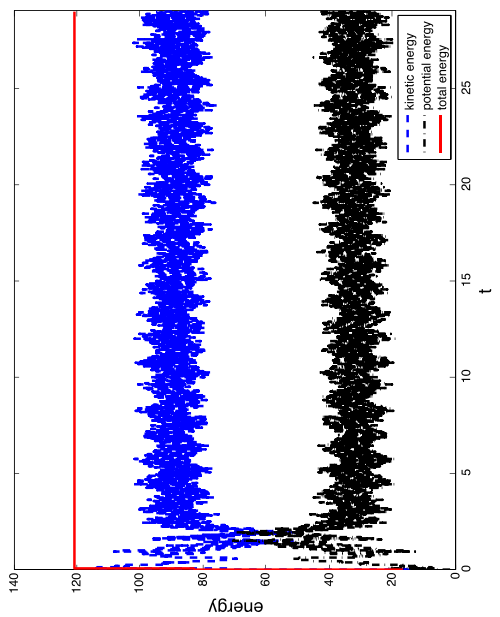

0
0
0
0
0
0
0
0
0
0
0
0
0
0
0
0
0
0
0
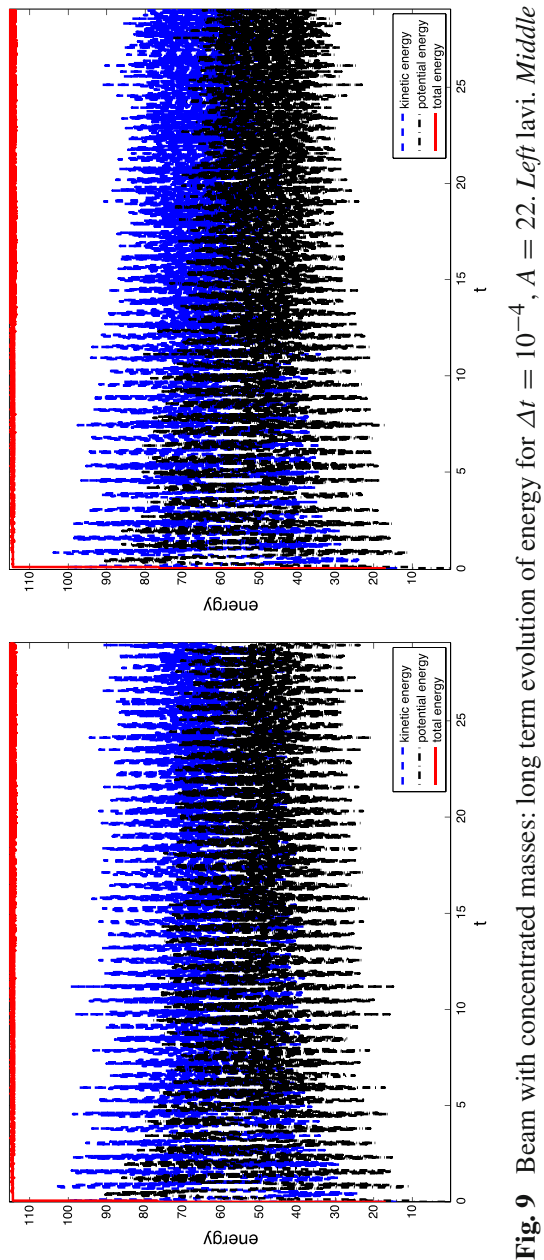


$$
\left(\varepsilon_{\mathrm{rel}}\right)_{a}^{j}=\frac{\left\|g_{a}^{j}-g_{\mathrm{ref}}\left(t^{j}, S_{a}\right)\right\|_{2}}{\left\|g_{\mathrm{ref}}\left(t^{j}, S_{a}\right)\right\|_{2}}
$$

each row containing the relative error at one time node for all spatial nodes. The relative error for a complete simulation result of the whole beam's motion during a time interval $[0, T]$ is given by

$$
\epsilon_{\mathrm{rel}}=\left\|\varepsilon_{\mathrm{rel}}\right\|_{F} \sqrt{\Delta t} \sqrt{\Delta l}
$$

where $\|\cdot\|_{F}$ is the Frobenius norm.

Convergence in time for fixed number of elements. For a fixed number of elements, we simulate the beam with decreasing time step $\Delta t$ to analyze the numerical convergence w.r.t. time grid refinement. Since the analytic solution is not known, we compute a reference solution with small time step for each method in the following way: the reference solution is computed with the same method, the same $\Delta l$, and $\Delta t=10^{-5}$. This is done for all element lengths. In Fig. 10, the relative error for the three methods is plotted logarithmically over the time step $\Delta t$ for each element length $\Delta l$. The convergence rates are approximately 1.6 for lavi and lgvi, and 2.3 for feem. Note that by fixing $\Delta l$, the convergence analysis corresponds to a convergence analysis for an ode-system w.r.t. $\Delta t$. For all three methods, we would expect a rate of 2.0, thus, our numerical results almost confirm this rate (also note that we have no real solution as reference which may lead to slightly different orders).

Convergence in space for fixed time step. For a fixed time step, we simulate the beam with decreasing element length $\Delta l$ to analyze the numerical convergence w.r.t. space grid refinement. The reference solution is computed with the same method, the same $\Delta t$, and $A=256$. This is done for all time steps. In Fig. 11, the relative error is plotted logarithmically over the space step $\Delta l$ for each time step $\Delta t$. The convergence rates are approximately 1.5 for lavi and lgvi, and 2.0 for feem.

Convergence in space and time simultaneously. Now we decrease the time and space step simultaneously in the same way. For each method, the reference solution is computed with the same method using $\Delta t=10^{-5}$ and $A=256$. This is done for $(\Delta t, A)=\left(1.6 \cdot 10^{-4}, 16\right),\left(8 \cdot 10^{-5}, 32\right),\left(4 \cdot 10^{-5}, 64\right),\left(2 \cdot 10^{-5}, 128\right)$. The relatives errors are given in Fig. 12. Since the space error dominates, the convergence rates behave in the same way as the convergence rates in space, and are thus approximately 1.5 for lavi and lgvi and 2.0 for feem.

\subsubsection{Differences between the methods}

Next we analyze the convergence behavior between different methods. The trajectories obtained by the three methods are computed for fixed $\Delta l$ and $\Delta t$. This is done for all time steps and for all element lengths. For $(\Delta t, A)=\left(1.6 \cdot 10^{-4}, 16\right),\left(8 \cdot 10^{-5}, 32\right)$, $\left(4 \cdot 10^{-5}, 64\right),\left(2 \cdot 10^{-5}, 128\right),\left(1 \cdot 10^{-5}, 256\right)$ the relative errors are plotted in Fig. 13 versus $\Delta t$ (versus space step $\Delta l$ gives the same pictures). The solution of one method 


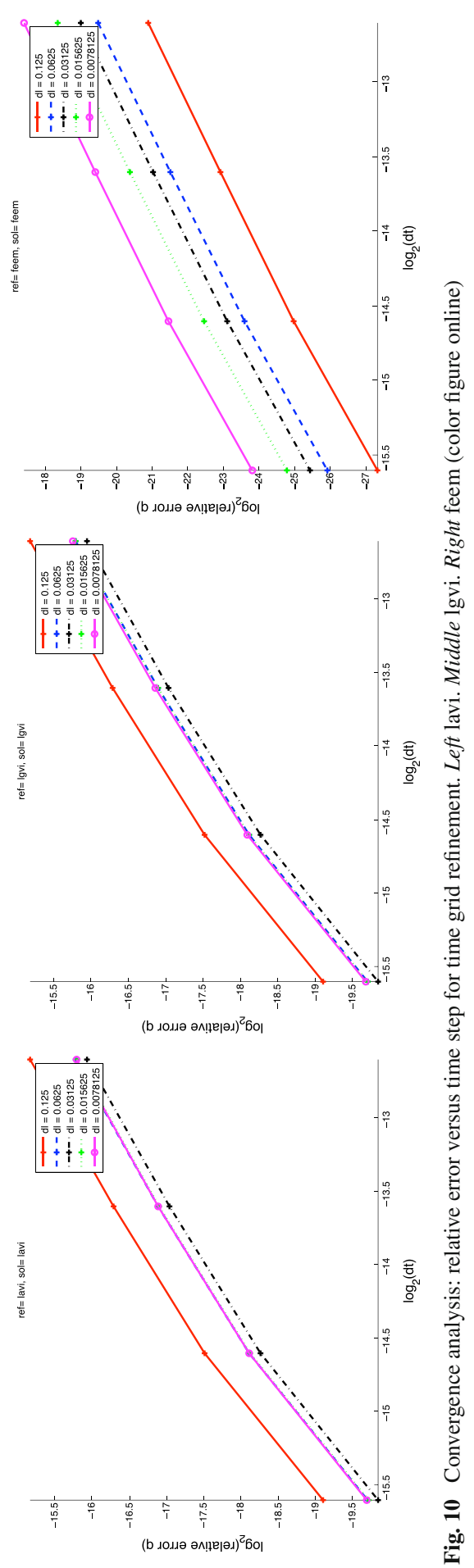




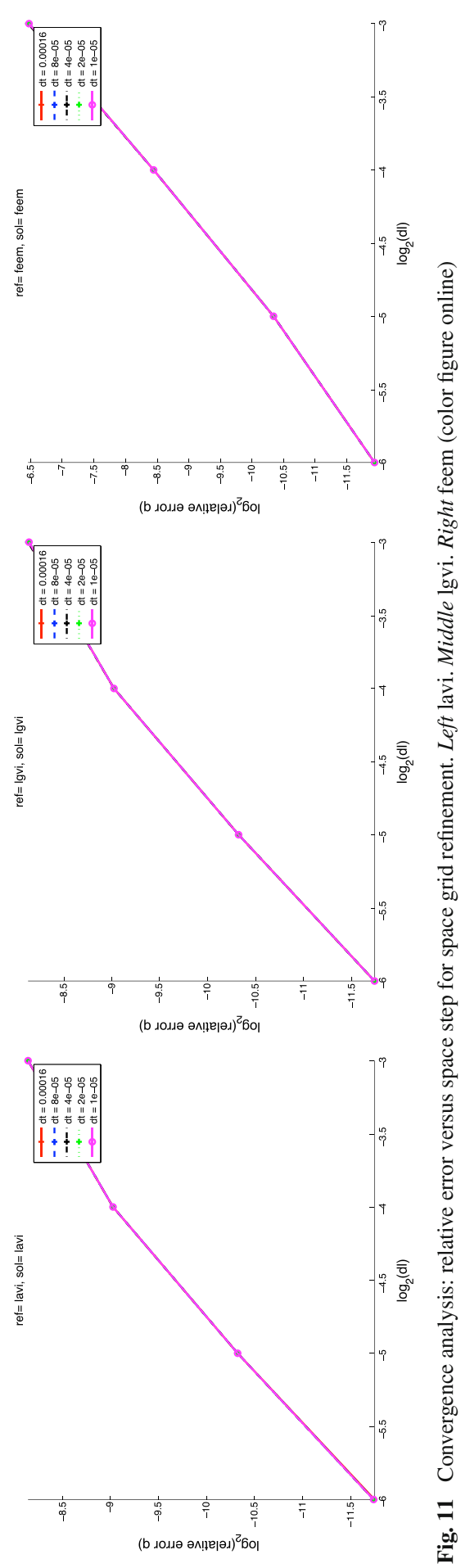




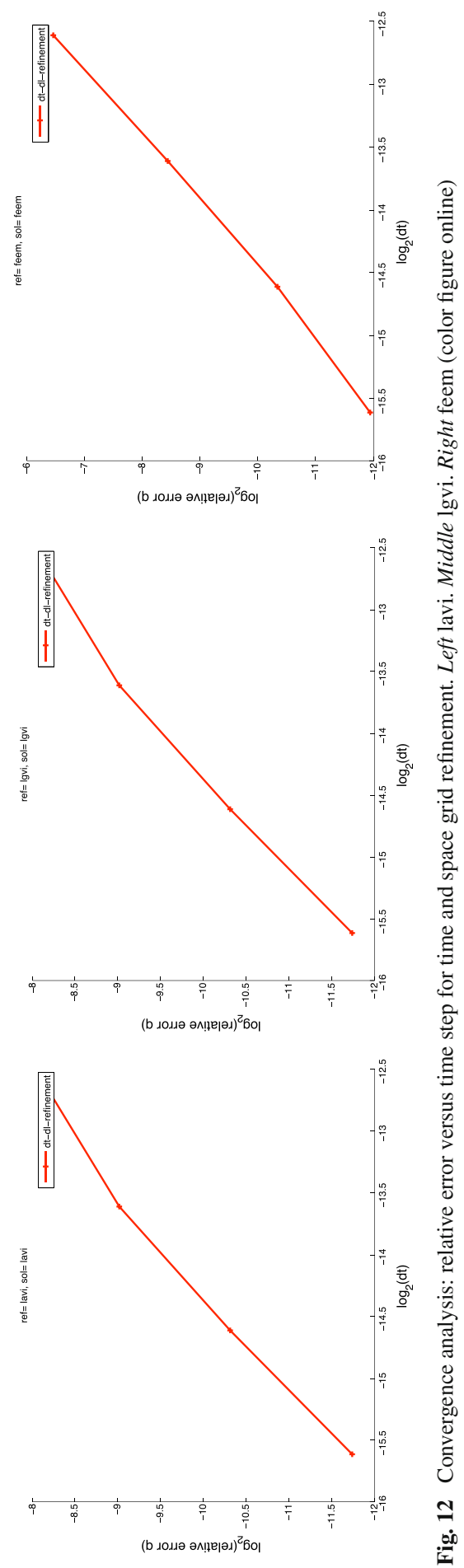




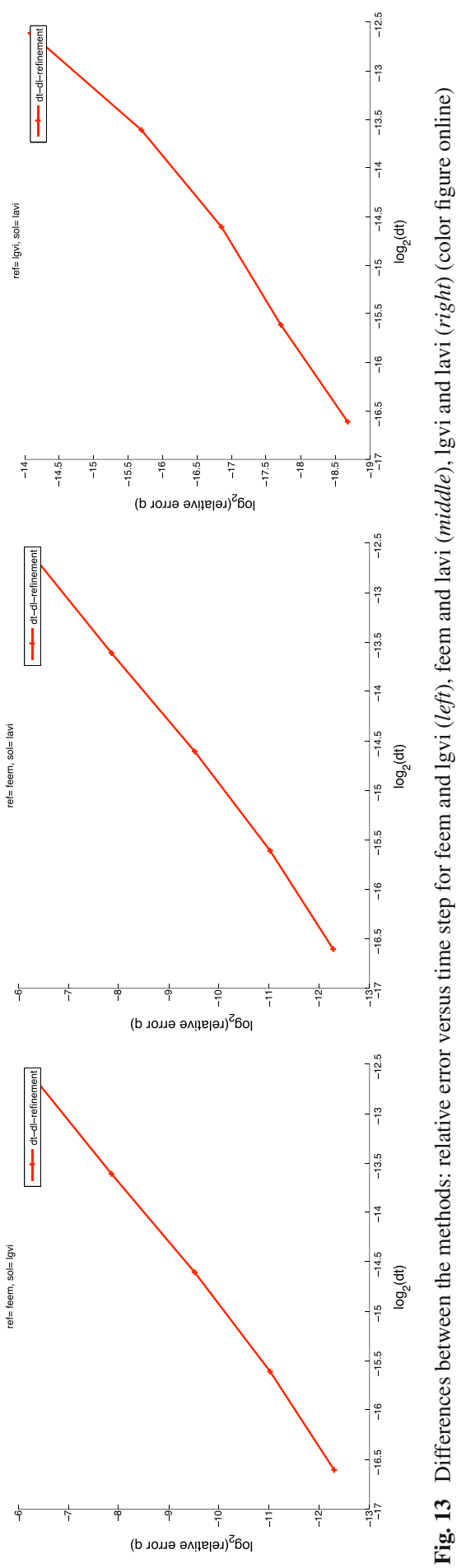



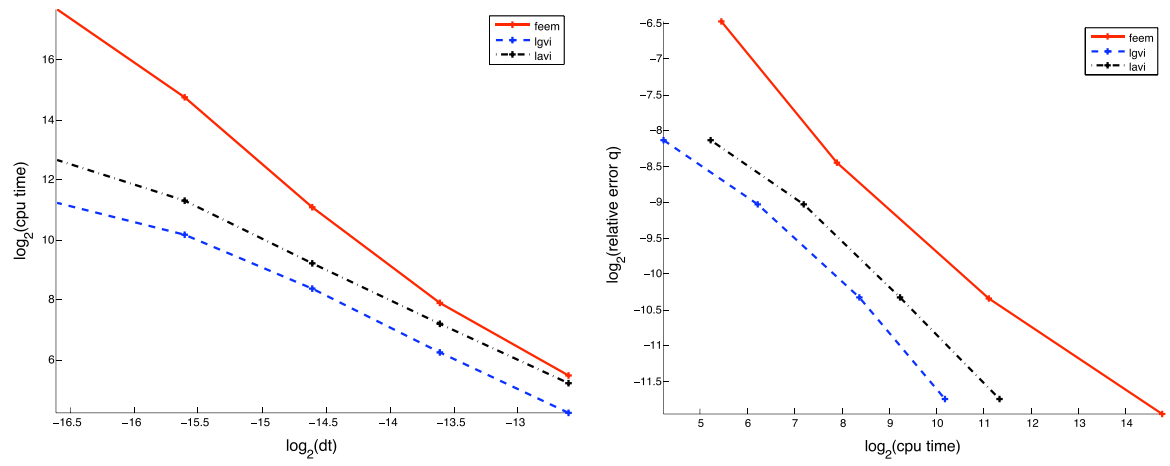

Fig. 14 Computational efficiency for each method. Left computational time versus time step. Right relative error versus computational time (color figure online)

does converge to the solution of another method with a rate of 1.6. lgvi and lavi lie much closer together than feem. This is to be expected, since they have the same space discretization and the same mass matrix whereas feem uses a different space discretization.

\subsubsection{Efficiency of each method}

We store the computational time for different discretizations in time and space for each method. In Fig. 14 (left), the computational time versus the time step (versus space step gives the same picture) is plotted logarithmically. The general trend is as excepted: for finer grids the computational time increases. lgvi and lavi are both faster than feem, lgvi is even slightly faster than lavi. However, note that feem is a fully implicit method (solving a nonlinear coupled system for the complete beam) whereas lgvi and lavi solve a (partly explicit) system for each node. For lavi and lgvi the computational time behaves approximately quadratic w.r.t. the time step (or space step, respectively), for feem the computational time behaves approximately cubic, especially during the first refinement steps. In Fig. 14 (right), the relative error versus the computational time is plotted logarithmically. The reference solutions for the computation of the relative error are chosen as in Sect. 4.3.1. Again, the general trend is as excepted: for more accurate approximations more computational time is required.

\subsubsection{Concluding remarks}

The numerical experiments illustrate that the two Lie group variational integrators yield quite similar simulation results, even for relatively coarse discretizations. Furthermore, the convergence rates are approximately of order 1.5 in space and in time. In contrast to that, the energy-momentum preserving time stepping scheme with finite elements in space shows space and time convergence rates of order 2.0. On coarse grids, the latter method's results differ from the first two methods' results substantially, while the results of all methods approach each other for grid refinement. Concerning the computational efficiency, the Lie group variational integrators are generally faster 
than the energy-momentum preserving time stepping scheme with finite elements, with the first Lie group integrator being the fastest.

Acknowledgments FD, TSR, and YW were partially supported by Swiss NSF Grant 200020-137704. TSR was partially supported by the government grant of the Russian Federation for support of research projects implemented by leading scientists, Lomonosov Moscow State University under the agreement No. 11.G34.31.0054. FGB was partially supported by a "Projet Incitatif de Recherche" contract from the Ecole Normale Supérieure de Paris and by the Swiss NSF Grant 200021-126802. SO was partially supported by the German Federal Ministry of Education and Research (BMBF) within the Leading-Edge Cluster "Intelligent Technical Systems OstWestfalenLippe" (it's OWL) and managed by the Project Management Agency Karlsruhe (PTKA).

\section{References}

1. Abraham, R., Marsden, J.E.: Foundations of Mechanics, 2nd edn, revised and enlarged. With the assistance of Tudor Ratiu and Richard Cushman. Benjamin/Cummings Publishing Co., Inc., Advanced Book Program, Reading, MA (1978)

2. Antman, S.S.: Kirchhoff's problem for nonlinearly elastic rods. Q. J. Appl. Math. 32, 221-240 (1974)

3. Antmann, S.S.: Nonlinear Problems in Elasticity. Springer, Berlin (1995)

4. Betsch, P., Menzel, A., Stein, E.: On the parametrization of finite rotations in computational mechanics: a classification of concepts with application to smooth shells. Comput. Methods Appl. Mech. Eng. 155, 273-305 (1998)

5. Betsch, P., Steinmann, P.: Frame-indifferent beam finite elements based upon the geometrically exact beam theory. Int. J. Numer. Methods Eng. 54, 1775-1788 (2002)

6. Betsch, P., Steinmann, P.: Constrained dynamics of geometrically exact beams. Comput. Mech. 31, 49-59 (2003)

7. Bobenko, A.I., Suris, Y.B.: Discrete time Lagrangian mechanics on Lie groups, with an application to the Lagrange top. Commun. Math. Phys. 204, 147-188 (1999a)

8. Bobenko, A.I., Suris, Y.B.: Discrete Lagrangian reduction, discrete Euler-Poincaré equations, and semidirect products. Lett. Math. Phys. 49, 79-93 (1999b)

9. Bottasso, C., Borri, M., Trainelli, L.: Geometric invariance. Comput. Mech. 29, 163-169 (2002)

10. Bou-Rabee, N., Marsden, J.E.: Hamilton-Pontryagin integrators on Lie groups Part I: introduction and structure-preserving properties. Found. Comput. Math. 9, 197-219 (2009)

11. Bou-Rabee, N., Owhadi, H.: Stochastic variational integrators. IMA J. Numer. Anal. 29, 421-443 (2008)

12. Brüls, O., Cardona, A.: On the use of Lie group time integrators in multibody dynamics. J. Comput. Nonlinear Dyn. 5(3), 031002 (2010). Special issue on Multi- disciplinary High-Performance Computational Multibody Dynamics, edited by Dan Negrut and Olivier Bauchau. doi:10.1115/1.4001370

13. Brüls, O., Cardona, A., Arnold, M.: Lie group generalized- $\alpha$ time integration of constrained flexible multibody systems. Mech. Mach. Theory 48, 1212-137 (2012)

14. Crisfield, M.A., Jelenić, G.: Objectivity of strain measures in the geometrically exact three-dimensional beam theory and its finite-element implementation. Proc. R. Soc. Lond. A 455, 1125-1147 (1999)

15. de León, M., Marrero, J.C., Martín de Diego, D.: Some applications of semi-discrete variational integrators to classical field theories. Qual. Theory. Dyn. Syst. 7(1), 195-212 (2008)

16. Demoures, F., Gay-Balmaz, F., Kobilarov, M., Ratiu, T.S.: Multisymplectic Lie group variational integrators for a geometrically exact beam in $\mathbb{R}^{3}$. Commun. Nonlinear Sci. Numer. Simul. 19(10), 3492-3512 (2014). http://arxiv.org/pdf/1403.5410v1

17. Dichmann, D.J., Li, Y., Maddocks, J.H.: Hamiltonian formulations and symmetries in rod mechanics. In: Mathematical Approaches to Biomolecular Structure and Dynamics, vol. 82, pp. 71-113. Springer, New York (1996)

18. Ellis, D., Gay-Balmaz, F., Holm, D.D., Putkaradze, V., Ratiu, T.S.: Symmetry reduced dynamics of charged molecular strands. Arch. Rat. Mech. Anal. 197(2), 811-902 (2010)

19. Fetecau, R.C., Marsden, J.E., Ortiz, M., West, M.: Nonsmooth Lagrangian mechanics and variational collision integrators. SIAM J. Appl. Dyn. Syst. 2(3), 381-416 (2003) 
20. Gay-Balmaz, F., Holm, D.D., Ratiu, T.S.: Variational principles for spin systems and the Kirchhoff rod. J. Geom. Mech. 1(4), 417-444 (2009)

21. Gay-Balmaz, F., Marsden, J.E., Ratiu, T.S.: Reduced variational formulations in free boundary continuum mechanics. J. Nonlinear Sci. 22(4), 463-497 (2012)

22. Gonzalez, O.: Time integration and discrete Hamiltonian systems. J. Nonlinear Sci. 6, 449-467 (1996)

23. Hairer, E., Lubich, C., Wanner, G.: Geometric Numerical Integration, Structure-Preserving Algorithms for Ordinary Differential Equations. Springer, Berlin (2006)

24. Ibrahimbegović, A., Frey, F., Kozar, I.: Computational aspects of vector-like parametrization of threedimensional finite rotations. Int. J. Numer. Methods Eng. 38, 3653-3673 (1995)

25. Ibrahimbegović, A., Mamouri, S.: Finite rotations in dynamics of beams and implicit time-stepping schemes. Int. J. Numer. Methods Eng. 41, 781-814 (1998)

26. Iserles, A., Munthe-Kaas, H.Z., Nørsett, S.P., Zanna, A.: Lie-group methods. Acta Num. 9, 215-365 (2000)

27. Jelenić, G., Crisfield, M.: Interpolation of rotational variables in non-linear dynamics of 3D beams. Int. J. Numer. Methods Eng. 43, 1193-1222 (1998)

28. Jelenić, G., Crisfield, M.: Geometrically exact 3D beam theory: implementation of a strain-invariant finite element for statics and dynamics. Comput. Methods Appl. Mech. Eng. 171, 141-171 (1999)

29. Jelenić, G., Crisfield, M.: Problems associated with the use of Cayley transform and tangent scaling for conserving energy and momenta in the Reissner-Simo beam theory. Commun. Numer. Methods Eng. 18, 711-720 (2002)

30. Jung, P., Leyendecker, S., Linn, J., Ortiz, M.: A discrete mechanics approach to Cosserat rod theory. Part 1: static equilibria. Int. J. Numer. Methods Eng. 85, 31-60 (2010)

31. Kane, C., Marsden, J.E., Ortiz, M., West, M.: Variational integrators and the Newmark algorithm for conservative and dissipative mechanical systems. Int. J. Numer. Methods Eng. 49(10), 1295-1325 (2000)

32. Kobilarov, M., Marsden, J.E.: Discrete geometric optimal control on Lie groups. IEE Trans. Robot. 27, 641-655 (2011)

33. Kobilarov, M., Marsden, J.E., Sukhatme, G.S.: Geometric discretization of nonholonomic systems with symmetries. Discrete Contin. Dyn. Syst. Ser. S 3(1), 61-84 (2010)

34. Lang, H., Linn, J., Arnold, M.: Multi-body dynamics simulation of geometrically exact Cosserat rods. Multibody Syst. Dyn. 25(3), 285-312 (2011)

35. Lang, H., Arnold, M.: Numerical aspects in the dynamic simulation of geometrically exact rods. Appl. Numer. Math. 62(10), 1411-1427 (2012)

36. Lee, T., Leok, M., McClamroch, N.H.: Dynamics of a 3D elastic string pendulum. In: Proceedings of the IEEE Conference on Decision and Control (2009)

37. Lee, T.: Computational geometric mechanics and control of rigid bodies. PhD Thesis, University of Michigan (2008)

38. Lew, A., Marsden, J.E., Ortiz, M., West, M.: Asynchronous variational integrators. Arch. Ration. Mech. Anal. 167(2), 85-146 (2003)

39. Lew, A., Marsden, J.E., Ortiz, M., West, M.: Variational time integrators. Int. J. Numer. Methods Eng. 60(1), 153-212 (2004)

40. Lew, A., Marsden, J.E., Ortiz, M., West, M.: An overview of variational integrators. In: Franca, L.P., Tezduyar, T.E., Masud, A. (eds.) Finite Element Methods: 1970's and Beyond, CIMNE, pp. 98-115 (2004)

41. Leyendecker, S., Betsch, P., Steinmann, P.: Objective energy-momentum conserving integration for the constrained dynamics of geometrically exact beams. Comput. Methods Appl. Mech. Eng. 195, 2313-2333 (2006)

42. Leyendecker, S., Betsch, P., Steinmann, P.: The discrete null space method for the energy consistent integration of constrained mechanical systems. Part III: flexible multibody dynamics. Multibody Syst. Dyn. 19, 45-72 (2008)

43. Leyendecker, S., Marsden, J.E., Ortiz, M.: Variational integrators for constrained dynamical systems. J. Appl. Math. Mech. 88(9), 677-708 (2008)

44. Leyendecker, S., Ober-Blöbaum, S.: A variational approach to multirate integration for constrained systems. In: Fisette, P., Samin, J.-C. (eds.) ECCOMAS Thematic Conference: Multibody Dynamics: Computational Methods and Applications, Brussels, Belgium, 4-7 July 2011 (2011)

45. Leyendecker, S., Ober-Blöbaum, S., Marsden, J.E., Ortiz, M.: Discrete mechanics and optimal control for constrained systems. Optim. Control Appl. Methods 31(6), 505-528 (2010) 
46. Marsden, J.E., Hughes, J.R.: Mathematical Foundations of Elasticity. Dover, New York (1994)

47. Marsden, J.E., Patrick, G., Shkoller, S.: Multisymplectic geometry, variational integrators, and nonlinear PDEs. Commun. Math. Phys. 199(2), 351-395 (1998)

48. Marsden, J.E., Pekarsky, S., Shkoller, S.: Discrete Euler-Poincaré and Lie-Poisson equations. Nonlinearity 12(6), 1647-1662 (1999)

49. Marsden, J.E., Ratiu, T.S.: Introduction to Mechanics and Symmetry. Springer, Berlin (1999)

50. Marsden, J.E., West, M.: Discrete mechanics and variational integrators. Acta Numer. 10, 357-514 (2001)

51. Moser, J., Veselov, A.P.: Discrete versions of some classical integrable systems and factorization of matrix polynomials. Commun. Math. Phys. 139, 217-243 (1991)

52. Ober-Blöbaum, S., Junge, O., Marsden, J.E.: Discrete mechanics and optimal control: an analysis. Control Optim. Calc. Var. 17(2), 322-352 (2011)

53. Reissner, E.: On one-dimensional finite strain beam theory: the plane problem. J. Appl. Math. Phys. 23, 795-804 (1972)

54. Reissner, R.: On a one-dimensional, large-displacement, finite-strain beam-theory. Stud. Appl. Math. 52, 87-95 (1973)

55. Romero, I., Armero, F.: An objective finite element approximation of the kinematics of geometrically exact rods and its use in the formulation of an energy-momentum scheme in dynamics. Int. J. Numer. Methods Eng. 54, 1683-1716 (2002)

56. Romero, I.: The interpolation of rotations and its application to finite element models of geometrically exact rods. Comput. Mech. 34, 121-133 (2004)

57. Shabana, A.: Dynamics of Multibody Systems. Cambridge University Press, Cambridge (1998)

58. Shabana, A., Yacoub, R.Y.: Three dimensional absolute nodal coordinate formulation for beam elements: theory. ASME J. Mech. Des. 123, 606-613 (2001)

59. Simo, J.C.: A finite strain beam formulation. The three-dimensional dynamic problem. Part I. Comput. Meth. Appl. Mech. Eng. 49, 79-116 (1985)

60. Simo, J.C., Marsden, J.E., Krishnaprasad, P.S.: The Hamiltonian structure of nonlinear elasticity: the material, spatial and convective representations of solids, rods and plates. Arch. Ration. Mech. Anal. 104, 125-183 (1988)

61. Simo, J.C., Vu-Quoc, L.: A three-dimensional finite-strain rod model. Part II: computational aspects. Comput. Meth. Appl. Mech. Eng. 58, 55-70 (1986)

62. Simo, J.C., Vu-Quoc, L.: On the dynamics in space of rods undergoing large motions-a geometrically exact approach. Comput. Meth. Appl. Mech. Eng. 66, 125-161 (1988)

63. Tao, M., Owhadi, H., Marsden, J.E.: Nonintrusive and structure preserving multiscale integration of stiff ODEs, SDEs, and Hamiltonian systems with hidden slow dynamics via flow averaging. Multiscale Model. Simul. 8(4), 1269-1324 (2010) 